\title{
Phenomenology and cosmology of an electroweak pseudo-dilaton and electroweak baryons
}

\author{
Bruce A. Campbell, ${ }^{a, b}$ John Ellis ${ }^{c, b}$ and Keith A. Olive ${ }^{d}$ \\ ${ }^{a}$ Department of Physics, Carleton University, \\ 1125 Colonel By Drive, Ottawa, Ontario K1S 5B6, Canada \\ ${ }^{b}$ TH Division, Physics Department, CERN, \\ CH-1211 Geneva 23, Switzerland \\ ${ }^{c}$ Theoretical Particle Physics and Cosmology Group, Physics Department, King's College London, \\ London, WC2R 2LS, U.K. \\ ${ }^{d}$ William I. Fine Theoretical Physics Institute, University of Minnesota, \\ 116 Church Street S.E., Minneapolis, MN 55455, U.S.A. \\ E-mail: campbell@physics.carleton.ca, John.Ellis@cern.ch, \\ olive@physics.umn.edu
}

ABSTRACT: In many strongly-interacting models of electroweak symmetry breaking the lowest-lying observable particle is a pseudo-Goldstone boson of approximate scale symmetry, the pseudo-dilaton. Its interactions with Standard Model particles can be described using a low-energy effective nonlinear chiral Lagrangian supplemented by terms that restore approximate scale symmetry, yielding couplings of the pseudo-dilaton that differ from those of a Standard Model Higgs boson by fixed factors. We review the experimental constraints on such a pseudo-dilaton in light of new data from the LHC and elsewhere. The effective nonlinear chiral Lagrangian has Skyrmion solutions that may be identified with the 'electroweak baryons' of the underlying strongly-interacting theory, whose nature may be revealed by the properties of the Skyrmions. We discuss the finite-temperature electroweak phase transition in the low-energy effective theory, finding that the possibility of a firstorder electroweak phase transition is resurrected. We discuss the evolution of the Universe during this transition and derive an order-of-magnitude lower limit on the abundance of electroweak baryons in the absence of a cosmological asymmetry, which suggests that such an asymmetry would be necessary if the electroweak baryons are to provide the cosmological density of dark matter. We revisit estimates of the corresponding spin-independent dark matter scattering cross section, with a view to direct detection experiments.

Keywords: Higgs Physics, Cosmology of Theories beyond the SM, Technicolor and Composite Models 


\section{Contents}

1 Introduction 1

2 The nonlinear effective electroweak and dilaton Lagrangian 3

2.1 Minimal violation of conformal symmetry 4

2.2 Non-zero anomalous dimensions 5

2.3 Anomalous loop-induced couplings 5

2.4 Precision electroweak data 8

$\begin{array}{lll}3 & \text { Electroweak baryons } & 9\end{array}$

4 The electroweak phase transition $\quad 11$

4.1 Finite-temperature corrections 11

$\begin{array}{lll}4.2 & \text { Critical temperature } & 12\end{array}$

$\begin{array}{lll}4.3 & \text { Tunnelling and supercooling } & 13\end{array}$

$\begin{array}{lll}4.4 & \text { Percolation } & 15\end{array}$

$\begin{array}{ll}4.5 \text { Confinement } & 17\end{array}$

$\begin{array}{lll}4.6 & \text { Electroweak baryon-to-entropy ratio } & 18\end{array}$

5 Electroweak baryons as dark matter 20

6 Detection of electroweak baryonic dark matter 22

$\begin{array}{lll}7 & \text { Summary } & 24\end{array}$

\section{Introduction}

The LHC has demonstrated the need for new physics in the electroweak symmetry-breaking sector.

A couple of inverse femtobarns of data recorded by each of CMS and ATLAS have sufficed to exclude a Standard Model Higgs boson at the 90\% CL or more between $\sim 130$ and $\sim 500 \mathrm{GeV}[1-3]$. As is well known, if $m_{H}>500 \mathrm{GeV}$, the electroweak symmetrybreaking sector becomes strongly-interacting at an energy scale $<1 \mathrm{TeV}$ [4-7], implying the emergence of new physics before that energy, which would also be required for compatibility with precision electroweak data [8,9]. As is also well known, if $m_{H}<130 \mathrm{GeV}$, the electroweak vacuum of the Standard Model is destabilized by loop corrections due to the top quark [10-12]. It might be argued that one could live with an electroweak vacuum that is metastable on a cosmological time scale, but most people might feel that this instability should be countered by the intervention of new physics, though its energy scale is rather 
uncertain. One way to stabilize the electroweak vacuum in the light Higgs case is lowenergy supersymmetry [13], though other mechanisms are possible. In addition to these low- and high-mass Standard Model Higgs cases, however, the LHC data are compatible with a third option for new physics, namely the appearance of a scalar Higgs-like state with suppressed couplings to Standard Model particles [14-20]. ${ }^{1}$

This paper is devoted to studying possible new physics in one generic scenario for such a third way, namely new, nearly conformal strong dynamics with a (relatively) light pseudodilaton that has (many) couplings proportional to those of a Standard Model Higgs boson, but potentially suppressed by some unknown factor, as occurs in several scenarios [14-20]. ${ }^{2}$ It is interesting to note that the most $(\sim 1.6 \sigma)$ significant fluctuation in the ATLAS/CMS Higgs combination is at $m_{H} \sim 146 \mathrm{GeV}$ with a suppressed strength $\sim$ half the expected signal in the Standard Model [1-3]. Such a 'less-Higgs' scenario is not renormalizable, and requires some model-dependent ultra-violet completion and hence new physics at the $\mathrm{TeV}$ scale. Our aim is to explore how much can be said about such a scenario without reliance on a specific model, and what information low-energy physics might be able to provide about this unknown ultraviolet completion.

A general framework for describing such a scenario is provide by nonlinear phenomenological Lagrangians. These were introduced in the 1960s to describe the low-energy interactions of pions [25-27], and their extension to include the pseudo-dilaton of approximate scale invariance of the strong interactions was developed shortly thereafter [28, 29]. A decade later, the nonlinear chiral Lagrangian formalism was adapted to describe Higgsless models of electroweak symmetry breaking [30]. This formalism has attracted renewed attention within the last decade, particularly in the framework of strongly-interacting models of electroweak symmetry breaking [31, 32]. Many of these feature a parametrically light pseudo-Goldstone boson of approximate scale invariance of the new high-scale strong interactions [33-41]. The use of a phenomenological Lagrangian to describe the interactions and other properties of such an electroweak pseudo-dilaton $[15,16]$ could be justified more readily than was ever the case for its conjectural counterpart in the conventional strong interactions, for which parametrical lightness could not be demonstrated and no convincing experimental candidate was ever found.

That said, the use of a phenomenological Lagrangian for approximate scale invariance of the strong interactions provided some useful insights, including the likelihood that the pseudo-dilaton would decay rapidly into pions, making it difficult to identify [29, 42], and the discovery of the canonical trace anomaly [43-45] that foreshadowed that of QCD and was the forerunner of later calculations of the Higgs decay rates into $\gamma \gamma$ and gluon pairs [46]. Some twenty years ago, we also used the phenomenological Lagrangian for approximate scale invariance to gain insight into the transition between quark-gluon and hadronic descriptions of QCD [47, 48], arguing that the chiral transition might be first order and using the Skyrme description $[49,50]$ of baryons as chiral solitons to argue that this transition should be identified with deconfinement.

\footnotetext{
${ }^{1}$ Or even, Heaven forfend, the absence of any Higgs-like state at all, an even more exciting scenario for new physics.

${ }^{2}$ Other scenarios are frequently considered, for example that the Higgs boson is a composite NambuGoldstone boson arising from the spontaneous breakdown of some higher-order chiral symmetry [21-24].
} 
In this paper, we recycle this approach to explore the phenomenology of scenarios with an electroweak pseudo-dilaton in generic approximately scale-invariant strongly-interacting models of electroweak symmetry breaking. Quite generally, the effective nonlinear phenomenological electroweak Lagrangian has Skyrmion solutions [49, 50], which may be interpreted as 'electroweak baryons', whose possible properties we discuss below. These properties could cast light on the new, high-scale strong dynamics, much as the quantum numbers of conventional baryons and the rate for $\pi^{0} \rightarrow 2 \gamma$ decay provided advance evidence for the colour degree of freedom in QCD. We also use the phenomenological Lagrangian for approximate scale invariance to gain insight into the nature of the finite-temperature electroweak phase transition in such a theory, ${ }^{3}$ which corresponds to 'pseudo-confinement'. We argue that this transition is likely to be first-order with substantial supercooling and subsequent percolation of bubbles of the true electroweak vacuum. Finally, exploiting this discussion of the electroweak phase transition, we revisit estimates of the possible cosmological relic abundance of such electroweak Skyrmion 'electroweak baryons' [53], and discuss their possible suitability as dark matter candidates [54]. We also revisit estimates of the spin-independent cross section for electroweak baryon scattering on conventional matter [55], with a view to direct dark matter detection experiments.

\section{The nonlinear effective electroweak and dilaton Lagrangian}

As is well known, the tree-level couplings of the Higgs boson of the minimal Standard Model to quarks and leptons (the bosons $W^{+} W^{-}$and $Z^{0} Z^{0}$ ) are directly proportional to their masses (squared), as would be the case for a dilaton, the conjectural Goldstone boson of spontaneously-broken scale invariance. In fact, the scale invariance of the Standard Model is broken explicitly by the coefficient of the quadratic term in the effective potential of the Standard Model, as well as by loop effects. The Higgs sector of the Standard Model is actually identical with the $\mathrm{SU}(2)$ linear $\sigma$ model [56], which was used as a prototype for spontaneous chiral symmetry breaking in what was subsequently discovered to be QCD. ${ }^{4}$

However, the linear $\sigma$ model is not the most general phenomenological low-energy Lagrangian for spontaneously-broken chiral symmetry, a rôle that is played by a non-linear chiral Lagrangian [25-27], in which there is no candidate for a dilaton and scale invariance is broken explicitly by the pion decay constant, $f_{\pi}$. However, approximate scale invariance can be introduced into the non-linear chiral Lagrangian by introducing a dilaton $\chi$ whose v.e.v. $V$ breaks scale invariance spontaneously $[28,29]$. The resulting non-linear Lagrangian is equivalent to the linear $\sigma$ model if $f_{\pi}=V$, but this is not the case in general, and the non-linear model provides a natural one-parameter generalization of the linear one, albeit a generalization that is non-renormalizable if $f_{\pi} \neq V$.

In a similar way, one may replace the Higgs sector of the Standard Model by a nonlinear Lagrangian for the Goldstone bosons that are eaten by the $W^{ \pm}$and $Z^{0}$, in which

\footnotetext{
${ }^{3}$ For a more complete discussion of more specific scenarios, see [51, 52].

${ }^{4} \mathrm{Just}$ as the approximate chiral symmetry of the strong interactions was a first clue that they might be described by a vector-gluon (gauge) theory [57], this analogy makes it tempting to conclude that the new, high-scale strong dynamics should also be based on a gauge theory.
} 
the rôle of $f_{\pi}$ is taken by the electroweak scale $v=246 \mathrm{GeV}$ but there is no scalar Higgs boson [30]. As in the case of the chiral Lagrangian, scale invariance may be (approximately) restored by introducing an electroweak (pseudo-)dilaton field $\chi[15-20] .{ }^{5}$ As we review below, at the tree level the dilaton couplings to Standard Model particles are similar to those of a conventional Higgs boson, but rescaled by a factor $v / V$. Explicit scale invariance breaking cannot be avoided, due to breaking by loop effects, but can be parametrized simply if this breaking is small, as might be the case in models with small breaking of scale symmetry such as walking technicolour. ${ }^{6}$ The resulting non-linear phenomenological Lagrangian provides a minimal generalization of the Standard Model Higgs sector within which, e.g., the results of searches for the Higgs boson may be interpreted more broadly.

For nonlinear realization of conformal symmetry, in the limit of small explicit breaking, the nonlinear $\mathrm{SU}(2) \times \mathrm{SU}(2) \rightarrow \mathrm{SU}(2)$ effective Lagrangian for the electroweak Goldstone bosons and the pseudo-dilaton may be written in the form

$$
\begin{aligned}
\mathcal{L}= & \frac{v^{2}}{4}\left(D_{\mu} U\right)\left(D^{\mu} U^{\dagger}\right)\left(\frac{\chi}{V}\right)^{2}+\frac{1}{2} \partial_{\mu} \chi \partial^{\mu} \chi \\
& -\mathcal{V}(\chi)-\Sigma_{f} m_{f}\left(\bar{f}_{L} f_{R} U+\text { h.c. }\right)\left(\frac{\chi}{V}\right)+\ldots,
\end{aligned}
$$

where $v=246 \mathrm{GeV}$ has the same value as the conventional Higgs v.e.v., $U$ is a $2 \times 2$ unitary matrix: $U U^{\dagger}=1$ that can be parametrized by 3 real degrees of freedom $\pi_{i}$, which can be identified with the Goldstone bosons 'eaten' by the $W^{ \pm}$and $Z^{0}$ bosons, $D_{\mu}$ is the conventional electroweak covariant derivative, $\chi$ is the dilaton field and $V$ is its v.e.v. The $f$ are Standard Model fermions (quarks and leptons), and the dots indicate higher-order terms in the effective Lagrangian, some of which we discuss in the following. It is apparent from (2.1) that the couplings of the pseudo-dilaton to gauge bosons and Standard Model fermions are related to those of the Standard Model Higgs boson by a simple multiplicative factor $v / V$.

There are, as well, a mass term and self-couplings for the dilaton $\chi$, encoded in the effective potential $\mathcal{V}(\chi)$. These are induced by renormalization and any other (small) explicit breaking of conformal invariance in the underlying dynamics that gives rise to the effective theory described by (2.1). Small explicit breaking may arise via unsuppressed operators that are almost marginal, or via operators that are far from marginality, but which enter the dynamics with small coefficients. We analyze each of these cases below.

\subsection{Minimal violation of conformal symmetry}

In the case where the operator $O$ generating explicit breaking of the conformal invariance is almost marginal (ie. when the dimension $\Delta_{O}$ of $O$ satisfies $\left|\Delta_{O}-4\right| \ll 1$ ), then $\mathcal{V}(\chi)$ is calculable to leading order in $\left|\Delta_{O}-4\right|$ :

$$
\mathcal{V}(\chi)=B \chi^{4}\left[\ln (\chi / V)-\frac{1}{4}\right]
$$

\footnotetext{
${ }^{5}$ The previous work whose spirit is most similar to ours is that of $[15,16]$, and we comment in a couple of places on specific aspects of [17-20].

${ }^{6}$ In the case of QCD, scale invariance is broken strongly by the trace anomaly associated with renormalization.
} 
The logarithmic form of the dilaton effective potential is related to the trace anomaly found in [58-61], and the coefficient $B$ is related to the pseudo-dilaton mass:

$$
m^{2}=4 B V^{2}
$$

\subsection{Non-zero anomalous dimensions}

In the case where the dilaton effective potential is induced by an operator in the electroweak symmetry-breaking sector which has a non-zero anomalous dimension $\gamma$, the effective potential in $(2.2)$ acquires corrections that are $\mathcal{O}(\gamma)[15,16]$. Even if the anomalous dimension $\gamma$ were not small, conformal symmetry would be approximate if the coefficient of the anomalous potential term were small, corresponding to a light pseudo-dilaton, in which case the effective potential would take the form [41]:

$$
\mathcal{V}(\chi)=\frac{m^{2} \chi^{4}}{\gamma V^{2}}\left[\frac{1}{4+\gamma}\left(\frac{\chi}{V}\right)^{\gamma}-\frac{1}{4}\right]+\mathcal{O}\left(\left(\frac{m^{2}}{V^{2}}\right)^{2}\right) .
$$

Defining $\hat{\chi} \equiv \chi-V$ and expanding (2.4) around $V$, we find the following expressions for the trilinear and quadrilinear $\hat{\chi}$ self-couplings:

$$
\begin{aligned}
& g_{3 \hat{\chi}}=(5+\gamma+\ldots) \frac{m^{2}}{V}, \\
& g_{4 \hat{\chi}}=\left(11+6 \gamma+\gamma^{2}+\ldots\right) \frac{m^{2}}{V^{2}} .
\end{aligned}
$$

Even in the case of violation of conformal symmetry by (almost) marginal operators with $\gamma \rightarrow 0$, discussed above, these couplings differ from those in the Standard Model by significant factors that are in principle measurable in future experiments, such as the highluminosity LHC [62] or CLIC [63].

Moreover, pseudo-dilaton/Higgs proportionality would also be violated if there are additional $\bar{f} f$ couplings with non-zero anomalous dimensions $\gamma_{f}$, which would be proportional to $(\chi / V)^{1+\gamma_{f}}$, as considered in [17-20]. In the absence of a theory of flavour, it is not apparent why such couplings should be flavour-diagonal in the same basis as the Yukawa couplings giving rise to the fermion masses $m_{f}$. On the other hand, any such couplings are constrained by upper limits on flavour-changing neutral interactions, so we assume here that they are negligible.

\subsection{Anomalous loop-induced couplings}

Another possible deviation from the $v / V$ proportionality rule concerns pseudo-dilaton couplings to massless gauge bosons $G$, namely gluons $g$ and photons $\gamma$. As is well known, the corresponding Standard Model Higgs couplings are generated by anomalous fermion triangle diagrams (and related diagrams for bosons) that vanish when they have small masses compared to $m_{H}$ [46], and are suppressed by the universal $v / V$ factor in the pseudodilaton case:

$$
\mathcal{L}_{G G}=\left[\frac{\alpha_{s}}{8 \pi} b_{s} G_{a \mu \nu} G_{a}^{\mu \nu}+\frac{\alpha_{e m}}{8 \pi} b_{e m} F_{\mu \nu} F^{\mu \nu}\right]\left(\frac{\chi}{V}\right) .
$$


However, any additional charged or coloured states that might appear in a stronglyinteracting dynamical theory underlying the pseudo-dilaton and the electroweak Goldstone bosons would alter the anomaly coefficients $b_{s, e m}[15,16]$, leading to an enhancement in the case of the pseudo-dilaton-gg coupling, or likely a suppression in the case of the pseudodilaton- $\gamma \gamma$ coupling, due to a partial cancellation with the Standard Model $W^{+} W^{-}$loops. ${ }^{7}$

The coefficient $b_{s}$ in (2.7) is normalized so that in the limit $m_{H} \ll m_{t}$ the one-loop contribution from the top quark $\rightarrow 2 / 3$ in the Standard Model, and this a good approximation for $m_{H / \chi} \lesssim m_{t}$. The CMS and ATLAS upper limits [1-3] on the production of a Standard Model-like Higgs boson relative to the Standard Model prediction with the conventional three generations can be rephrased as an upper limit on $v / V$, and hence a lower limit on $V$ as a function of the dilaton mass $m$, as indicated by the solid red line in figure 1 , where we see that the LHC already imposes the limit $V \gtrsim 400 \mathrm{GeV}$ in some ranges of $m$. We recall that the most significant fluctuation in the ATLAS/CMS combination is a $1.6 \sigma$ fluctuation at $m_{H} \sim 146 \mathrm{GeV}$, with a strength $\sim 1 / 2$ that expected in the Standard Model [1-3].

Any additional heavy quark would also contribute $\Delta b_{s}=2 / 3$, so that a fourthgeneration doublet would approximately triple the value of $b_{s}$ and hence multiply the cross section for $g g \rightarrow H$ or $\chi$ by a factor of about 9 . However, this enhancement would be reduced for $m_{t} \lesssim m_{H / \chi} \lesssim 1000 \mathrm{GeV}$, since the top quark contribution yields a contribution to $\left|b_{s}\right|>2 / 3$ for this range of $m_{H / \chi}{ }^{8}$ On the other hand, since $b_{s} \rightarrow 0$ as $m_{t} / m_{H / \chi} \rightarrow 0$, the top quark contribution to $b_{s}$ will decrease to $\left|b_{s}\right|<2 / 3$ for $m_{H / \chi} \gg m_{t}$ so that if $m_{t} \ll m_{H / \chi} \lesssim 1000 \mathrm{GeV}$ the enhancement factor due to a fourth generation could exceed 9, depending on the masses of the fourth-generation quarks. The searches for a Standard Model-like Higgs boson at the LHC has already excluded a naive four-generation extension of the Standard Model over a large range of Higgs masses [1-3]. However, a four-generation scenario could be revived in a pseudo-dilaton theory, since the potential enhancement in $\sigma(g g \rightarrow H)$ could be compensated by the universal $(v / V)^{2}$ factor in the production rate. Figure 1 displays as a dashed blue line the 95\% CL lower limit on $V$ obtained from the CMS and ATLAS upper limits on a four-generation extension of the Standard Model given in $[1-3]$. For any given value of the dilaton mass $m$, we use the more sensitive of the ATLAS and CMS limits: this is provided by CMS for $m \leq 400 \mathrm{GeV}$ and by ATLAS for $m>400 \mathrm{GeV}$. We see that in the four-generation case the LHC already imposes the limit $V \gtrsim 800 \mathrm{GeV}$ in some ranges of $m$, and approaches $1 \mathrm{TeV}$ for $m \sim 220 \mathrm{GeV}$.

The coefficient $b_{e m}$ in (2.7) takes the following value in the Standard Model:

$$
b_{e m}=\frac{4}{3} \Sigma_{f} Q_{f}^{2}-7
$$

in the limit of small $m_{H / \chi},{ }^{9}$ where the first term in (2.8) includes a sum over all heavy fermions, with the quark contributions acquiring a colour counting factor of 3 , the second

\footnotetext{
${ }^{7}$ Specific contributions to these coefficients arise from techniquarks in the scenario of ref. [17-20].

${ }^{8}$ We recall that the imaginary part of $b_{s} \neq 0$ for $m_{H / \chi}>2 m_{t}$.

${ }^{9}$ For the generalization to non-zero $M_{H / \chi}$ see [64].
} 


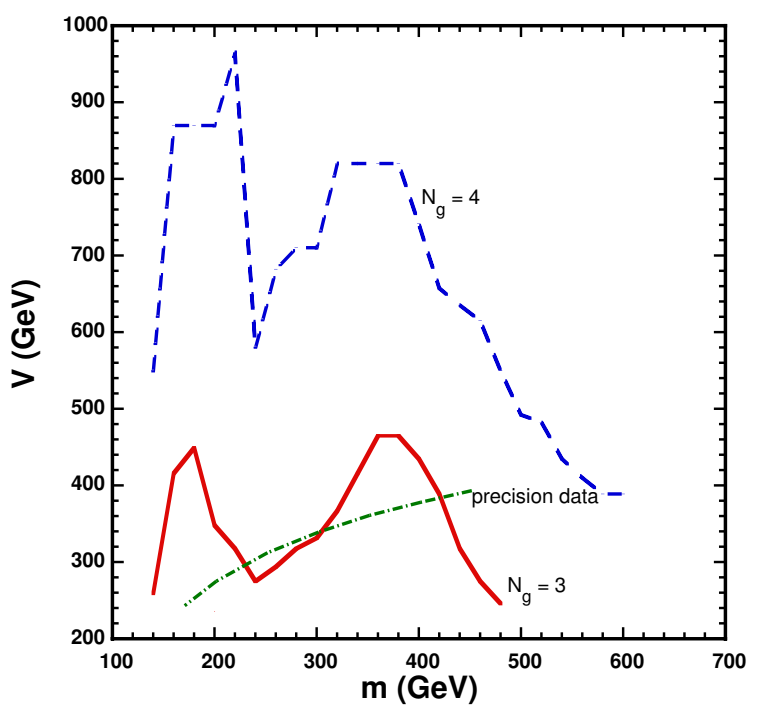

Figure 1. Lower limits on the pseudo-dilaton v.e.v. $V$ as functions of the its mass $m$, as obtained from an illustrative combination of the 95\% ATLAS and CMS upper limits on a Standard Model-like Higgs boson relative to the Standard Model with three generations (solid red line), and as obtained from the more sensitive of the ATLAS and CMS limits on the Standard Model with four generations (dashed blue line) [1-3]. Also shown, as a green dash-dotted line, is the lower limit on $V$ as a function of $m$ provided by precision electroweak data, estimated using (2.10) and the results of [9].

term in (2.8) is due to the $W^{ \pm},{ }^{10}$ and we note that the fermion and $W^{ \pm}$contributions have opposite signs. The terms in (2.8) are enhanced in the neighbourhoods of the thresholds where $m_{H / \chi} \sim 2 m_{t}, 2 m_{W}$, respectively, and decrease for larger $m_{H / \chi}$. As a result, $\Gamma(H / \chi \rightarrow \gamma \gamma)$ is strongly suppressed for $m_{H / \chi} \sim 2 m_{t}$. The effect of additional fermions would be to decrease the magnitude of $b_{e m}$ for small $m_{H / \chi}$, but the magnitude might be increased for larger masses, depending on the heavier quark masses. Hence the interpretation of the appearance (or absence) of a $\gamma \gamma$ signal would be ambiguous in a pseudo-dilaton

\footnotetext{
${ }^{10}$ The validity of this calculation has recently been questioned in two papers $[65,66]$ in which the unitary gauge was used to arrive at a different result. We recall that individual unitary-gauge Feynman diagrams are quartically divergent, so that great care must be taken to obtain the correct finite result. The relation of unitary-gauge and $R_{\xi}$-gauge calculations was discussed in [67], where it was pointed out that U-gauge integrals are formally equal to those in the $R_{\xi}$ gauge in the limit $\xi \rightarrow 0$, but that this equivalence is valid only if the limit is taken after integration over the Feynman parameters. It is known that, if sufficient care is not taken with the order of limits or in the definitions of loop momenta, the $U$ gauge may yield incorrect finite results. We also note that, in general, regularization is necessary even in calculations such as this that yield a finite result, and that neglecting regularization may yield an incorrect finite result. The result (2.8) has been verified in many independent calculations. Therefore, we do not regard the calculation of $[65,66]$ as evidence of a problem with dimensional regularization, which has given many correct results in all sectors of the Standard Model. The papers [65, 66] also contain incorrect remarks about the decoupling theorem and the trace anomaly. For recent refutations of the conclusions of [65, 66], see [68-72], where issues in the treatment of divergent integrals of the Goldstone (longitudinal $W^{ \pm}$) modes and decoupling are discussed. J.E. thanks Sasha Belyaev, David Broadhurst, Mary K. Gaillard, Dimitri Nanopoulos and Douglas Ross for discussions on these points.
} 
theory. On the other hand, the search for a $\gamma \gamma$ signal is currently not important for $M_{H} \gtrsim 130 \mathrm{GeV}$, the range displayed in figure 1, and we do not discuss it further here.

Finally, note that because any extra heavy (as yet undiscovered) coloured fermions, such as new fourth-generation quarks, or coloured states in the ultraviolet completion of the electroweak symmetry breaking sector, could produce an enhancement in $\sigma(g g \rightarrow H)$ that might compensate the universal $(v / V)^{2}$ suppression of the production rate, the observation of an $H / \chi$ state at a rate consistent with that of $H$ production in the Standard Model would not, by itself, prove that such a state was the Standard Model $H$, rather than a $\chi$. Furthermore, since the couplings of both the $H$ and $\chi$ to massive final states (which dominate the decay width) are proportional to mass, the measurement of the relative branching ratios to massive final states would not resolve this ambiguity. However, this ambiguity could be resolved in two ways.

One is by a measurement of the decay width of the state. Since in both cases the dominant contributions to the decay width are massive states, and the only difference in the $\chi$ couplings to the massive states, as opposed to an $H$, is the universal suppression by $v / V$, the decay width of a $\chi$ will, to good approximation, be the same as the Standard Model decay width of an $H$ of the same mass, but reduced by a factor $(v / V)^{2}$. So, if the resonance observed is at a mass at which the Standard Model predicts an observable decay width, measurement of the decay width at its predicted value would be evidence that the state was indeed a Standard Model Higgs. Conversely, if the decay width is measured to be smaller than the Standard Model prediction (or appears unmeasurably small) then one will have established that the state is not a Standard Model Higgs. Further, if the state is a pseudo-Dilaton $\chi$, and the decay width can be measured, that measurement will determine the ratio $v / V$. For $H / \chi$ states of moderately large masses, one of the dominant decay modes is to a $Z^{0} Z^{0}$ final state, and the decay chain $H / \chi \rightarrow Z^{0} Z^{0} \rightarrow \ell^{+} \ell^{-} \ell^{+} \ell^{-}$provides a powerful technique for the measurement of the decay width, and hence the identification of the state as an $H$ or a $\chi$.

A second way to resolve the $H / \chi$ is by disentangling the $g g$ fusion production mechanism from others, such as $W^{+} W^{-}$fusion and/or production in association with a $W^{ \pm}, Z^{0}$ or $\bar{t} t$ pair. In each of these cases, the production cross section is suppressed by the same factor $(v / V)^{2}$. This approach to $H / \chi$ discrimination would work better for a lighter state, whereas measuring the total decay width is easier for a more massive state.

\subsection{Precision electroweak data}

Before leaving this section, we discuss briefly the potential impact of the constraints imposed by precision electroweak data via determinations of the $S$ and $T$ vacuum polarization parameters $[73,74]$. The contributions of pseudo-dilaton loops are proportional to those from a conventional Higgs boson, but scaled by factors $(v / V)^{2}$ :

$$
\begin{aligned}
& \Delta S(\chi)=\left(\frac{v}{V}\right)^{2} \frac{1}{12 \pi} \ln \left(\frac{m^{2}}{m_{Z}^{2}}\right)+\ldots \\
& \Delta T(\chi)=-\left(\frac{v}{V}\right)^{2} \frac{3}{16 \pi \cos \theta_{W}^{2}}\left(\frac{m^{2}}{m_{Z}^{2}}\right)+\ldots
\end{aligned}
$$


where the dots denote terms that do not depend logarithmically on the dilaton mass. Standard Model fits to the precision electroweak data are compatible with a Higgs mass $\sim m_{Z}$, and hence a negligible contribution to $S$ and $T$ from the electroweak symmetry-breaking sector. The potential suppressions of the dilaton contributions by factors $(v / V)^{2}$ open the possibility that even a relatively heavy dilaton could be compatible with the precision electroweak data. Specifically, figure 10 of [9] shows that the pseudo-dilaton contributions to $S$ and $T(2.10)$ would lie within the present experimental upper bounds even for $m \sim 1 \mathrm{TeV}$, if $(v / V)^{2} \sim 1 / 10$. We display in figure 1 as a green dash-dotted line the lower limit on $V$ as a function of $m$ obtained using the forms (2.10) of the loop corrections and the analysis of [9] that found $m_{H}<169 \mathrm{GeV}$ at the $95 \% \mathrm{CL}$ in the Standard Model, i.e., when $V=v$, assuming three generations. We see that this gives a lower bound on $V$ that is weaker than the direct Higgs searches in the three-generation case (red line) over much of the range displayed. However, the limit from precision electroweak data is stronger than that from the direct Higgs searches in at large $m$, and is competitive in an intermediate range of $m .^{11}$

Nevertheless, a full discussion of the precision electroweak constraints requires a better understanding of the other contributions to $S$ and $T$ in a strongly-interacting theory, which are quite model-dependent. For example, a massive $\rho$-like vector resonance with self-coupling $g_{\rho} \epsilon_{a b c} \partial_{\mu} \rho_{\nu}^{a} \rho_{\mu}^{b} \rho_{\nu}^{c}$ would contribute [76-79]

$$
\Delta S(\rho)=\frac{4 \pi}{g_{\rho}^{2}}
$$

and other contributions are to be expected in any specific model. Therefore, it may be premature to conclude that the precision electroweak excludes any region of the pseudo-dilaton parameter space at the present level of understanding.

\section{Electroweak baryons}

If the searches for a Higgs boson do indeed discover a pseudo-dilaton, particle physics will be in a situation reminiscent of, but rather different from, that of strong-interaction physics in the 1960s. The similarity is that one has an effective low-energy theory but is ignorant of its ultraviolet completion and short-distance structure. In the 1960s, the approximate chiral symmetry of the low-energy effective theory motivated the suggestion that the underlying theory might be a gauge theory, and supplementary information, such as the rate for $\pi^{0} \rightarrow \gamma \gamma$ decay and the success of the quark model in describing baryons, in particular, suggested that the gauge group should be the SU(3) of colour, i.e., QCD. In the present case, we have less information. We have an effective low-energy Lagrangian, its chiral symmetry suggests that the underlying theory is a gauge theory, and the LHC is providing constraints on the pseudo-dilaton couplings. However, we currently have no analogue of the phenomenological success of the quark model to guide us towards the nature of the dynamics of any underlying theory.

\footnotetext{
${ }^{11}$ We do not discuss here the evaluation of the precision electroweak limit in the four-generation case, as it requires some supplementary hypotheses on the spectrum of fourth-generation quarks and leptons.
} 
However, as we discuss below, relevant experimental information may be provided by 'electroweak pseudo-baryons', which arise as Skyrmion solutions of the nonlinear effective electroweak Lagrangian. Their existence is 'inevitable' but their properties are modeldependent, and discovering any such states and measuring their properties would provide valuable insights into the underlying theory. ${ }^{12}$

It is well known that the nonlinear $\mathrm{SU}(3) \times \mathrm{SU}(3) \rightarrow \mathrm{SU}(3)$ and $\mathrm{SU}(2) \times \mathrm{SU}(2)$ $\rightarrow \mathrm{SU}(2)$ chiral Lagrangians of QCD have topological soliton solutions [49, 50, 81] called Skyrmions, as long as there is a higher-order 'Skyrme term':

$$
\mathcal{L}_{\mathcal{S}}=\frac{1}{32 e^{2}} \operatorname{Tr}\left[\left(D_{\mu} U\right) U^{\dagger},\left(D_{\nu} U\right) U^{\dagger}\right]^{2} .
$$

These solitons appear because $\pi_{3}(\mathrm{SU}(2))=\mathrm{Z}$, and have integer baryon number

$$
B=\frac{1}{24 \pi^{2}} \int d^{3} x \epsilon^{i j k} \operatorname{Tr}\left[\left(U^{-1} \partial_{i} U\right)\left(U^{-1} \partial_{j} U\right)\left(U^{-1} \partial_{k} U\right)\right] .
$$

In the case of the nonlinear $\mathrm{SU}(3) \times \mathrm{SU}(3) \rightarrow \mathrm{SU}(3)$ theory the effective Lagrangian contains a Wess-Zumino term $N \Gamma$, where $N$ is an integer, and the lowest-lying $B=1$ baryon is a fermion (boson) with $I=J=1 / 2(I=J=0)$ if $N$ is odd (even). If the underlying strongly-interacting theory is a non-Abelian $\mathrm{SU}(\mathrm{N})$ gauge theory, $N$ is identified with the number of colours, and in QCD the $B=1$ baryon is necessarily a fermion because $N=3$. In the case of the nonlinear $\mathrm{SU}(2) \times \mathrm{SU}(2) \rightarrow \mathrm{SU}(2)$ theory, there is no Wess-Zumino term, and the $B=1$ baryon is not necessarily a fermion. Nevertheless, the topology accommodates this possibility, since $\pi_{4}(\mathrm{SU}(2))=\mathrm{Z}_{2}[81]$.

The above discussion can be taken over intact to the case of the scale-invariant electroweak $\times \mathrm{SU}(2) \rightarrow \mathrm{SU}(2)$ chiral Lagrangian. The only difference is the appearance of a dilaton-dependent factor in the leading-order term in the chiral Lagrangian (but not the Skyrme term (3.1)), which does not affect the topological properties of the theory. Indeed, a $B=1$ Skyrmion solution of the field equations for the dilaton-dependent $\mathrm{SU}(3) \times \mathrm{SU}(3)$ $\rightarrow \mathrm{SU}(3)$ chiral QCD Lagrangian has been exhibited in [82], with the same qualitative features as in the dilaton-free theory. We therefore conclude that the (approximately) scale-invariant nonlinear electroweak Lagrangian possesses Skyrmion solutions with nonzero electroweak pseudo-baryon number $\mathcal{B} \neq 0$, which may be either baryons or fermions, depending on the nature of the underlying strongly-interacting theory.

As pointed out in [81], there are other exotic possibilities for the electroweak baryon quantum number, which should also be considered in the absence of information about the ultraviolet completion of the effective nonlinear Lagrangian. For example, if the underlying strongly-interacting theory is based on an $\mathrm{SO}(\mathrm{N})$ gauge theory, no distinction is possible between baryons and antibaryons, i.e., $\mathcal{B}$ is a $\mathrm{Z}_{2}$ quantum number. In this case, baryons are produced in pairs and two baryons may annihilate into $N$ mesons. Another, more exotic possibility arises if the underlying gauge theory is of $\mathrm{Sp}(\mathrm{N})$ type. In this case there are no stable baryons at all, and any wannabe baryon could decay directly into $N$ mesons.

\footnotetext{
${ }^{12}$ For related studies in 'Little Higgs' models, see [80].
} 
It has been suggested recently that the ultraviolet completion of the electroweak symmetry-breaking sector of the Standard Model might be via classical field configurations, dubbed 'classicalons' [83, 84]. In the case of QCD, the ultraviolet completion of the low-energy chiral theory involves an infinite series of resonant states, most of which are unstable against hadronic decays. The only states that are stable against hadronic decays are the lowest-lying baryons. From the point of view of this discussion, that is because they are the lowest states in a non-trivial topological sector. Indeed, in QCD these are the only known non-trivial semi-classical field configurations. However, they do not play an important rôle in unitarizing $\pi \pi$ scattering. As discussed above, though, they do encode key features of the underlying theory. In a similar way, the Skyrmions of the electroweak theory may encode important aspects of the dynamic underlying electroweak symmetry breaking that is not determined by the effective low-energy Lagrangian alone. However, they would not provide a realization of the 'classicalon' suggestion.

\section{The electroweak phase transition}

We now discuss the behaviour of the pseudo-dilaton theory at finite temperature, $T$, focusing in particular on the electroweak phase transition in this model. ${ }^{13}$ To this end, we first discuss the $T$ dependences of its three parameters with non-zero mass dimensions, namely $v, V$ and $m$, whose leading corrections are in general of the forms $\mathcal{O}\left(T^{2} / v^{2}, T^{2} / V^{2}\right)$.

\subsection{Finite-temperature corrections}

Since $v$ appears in the nonlinear electroweak Lagrangian in the same way as $f_{\pi}$ in the much-studied nonlinear pion Lagrangian of QCD, the leading $v$-dependent temperature corrections to $v$ itself are known from studies of that theory $[85,86]$ :

$$
v(T, v)=v \times\left[1-\frac{\mathcal{N} T^{2}}{24 v^{2}}+\mathcal{O}\left(\frac{T^{4}}{v^{4}}\right)\right],
$$

where $\mathcal{N}$ is the number of electroweak 'pseudo-flavours'. In the minimal realization of the Standard Model with an $\mathrm{SU}(2) \times \mathrm{SU}(2) \rightarrow \mathrm{SU}(2)$ structure, $\mathcal{N}=2$, but one could imagine embedding the theory in a larger structure with more 'pseudo-pions', in which case $\mathcal{N}>2$. In QCD, the leading $T / v$-dependent corrections to $\langle 0|\bar{q} q| 0\rangle$ have also been calculated, and are of the form $\left[1-\left(\mathcal{N}^{2}-1\right) T^{2} /\left(12 \mathcal{N} v^{2}\right)+\mathcal{O}\left(T^{4} / v^{4}\right)\right][85,86]$. Numerical evaluations suggest that $v(T, v)$ and $\langle 0|\bar{q} q| 0\rangle$ do not vanish for $T<2 v$, but the $T / v$ expansion is in principle valid only in the limit $T \ll v$, so this conclusion should be taken with a pinch of salt. Moreover, since the kinetic term for the Goldstone bosons in the nonlinear effective electroweak Lagrangian also has a factor $\chi^{2} / V^{2}$, there is also a finite-temperature correction to $v$ that are $\propto T^{2} /\left(12 V^{2}\right)$. The leading contributions to the finite-temperature dilaton effective potential are also well known:

$$
\delta \mathcal{V}(T, \chi)=\frac{B}{12} \chi^{2}\left[\ln \left(\frac{\chi}{V}\right)+\frac{1}{3}\right] T^{2}+\ldots,
$$

where the dots indicate terms that are of higher order in $T / V$.

\footnotetext{
${ }^{13}$ For an early study of technicolour cosmology, see [53], and for a more recent discussion see [51, 52]. For a more closely related recent discussion of cosmology in a nearly conformal model, see [87, 88].
} 


\subsection{Critical temperature}

The finite-temperature correction (4.2) does not alter the fact that the minimum of the effective potential is at $\langle\chi\rangle \neq 0$. However, the preferred finite-temperature vacuum is found by minimizing the free energy

$$
F \equiv-P+\mathcal{V}(\chi)+\delta \mathcal{V}(T, \chi)
$$

where

$$
P=\left(N_{B}+\frac{7}{8} N_{F}\right) \frac{\pi^{2}}{90} T^{4}
$$

for $N_{B}$ massless bosons and $N_{F}$ massless fermions, and hereafter we denote $\mathcal{N} \equiv N_{B}+$ $\frac{7}{8} N_{F}$. The finite-temperature electroweak phase transition is driven by the difference between (4.4) in the high-temperature vacuum with $\langle\chi\rangle=0$ and the low-temperature vacuum with $\langle\chi\rangle=V$.

The contributions of the Standard Model particles are the same in both states: the difference arises from the numbers of degrees of freedom in the electroweak symmetry-breaking sector above and below the transition temperature. In the low-temperature theory, this is simply $N_{B}=4$ for the pseudo-dilaton and the longitudinal polarization states of the $W^{ \pm}$ and $Z^{0}$. If the high-energy theory were a parity-conserving $\mathrm{SU}(\mathrm{N})$ gauge theory with $N_{f}$ multiplets of fermions in the fundamental representation, it would contribute

$$
\Delta \mathcal{N}=2\left(N^{2}-1\right)+\frac{7}{2}\left(N \times N_{f}\right)
$$

If this theory were to be approximately conformal, one would have $N_{f}=\mathcal{O}(11 N / 2)$, and hence

$$
\Delta \mathcal{N} \sim \frac{85}{4} N^{2}-6
$$

Substituting into (4.4), it is clear that, for any plausible $\mathcal{N}$, the difference between the pressures in the symmetric and broken phases is

$$
\Delta P=\Delta \mathcal{N} \frac{\pi^{2}}{30} T^{4} \sim \mathcal{O}(\text { few }) \pi^{2} T^{4} .
$$

This is a useful order of magnitude to keep in mind in the following discussion - the exact numerical coefficient does not play an essential rôle.

The critical temperature, $T_{c}$, is calculated by equating $F$ in the symmetric and broken phases:

$$
\Delta P=-\mathcal{V}\left(\chi_{T}\right)-\delta \mathcal{V}\left(T, \chi_{T}\right)
$$

where $\chi_{T}$ denotes the value of $\chi$ in the broken phase at finite-temperature. Neglecting $\delta \mathcal{V}(T, \chi)$, so that $\chi_{T} \sim V$, and using $\mathcal{V}=-(B / 4) V^{4}$ at the minimum in the broken phase and recalling that $m^{2}=4 B V^{2}$, one has

$$
T_{c}=\mathcal{O}\left(\frac{1}{2 \sqrt{\pi}}\right) \sqrt{m V}+\ldots \simeq 0.28 \sqrt{m V} .
$$


At this temperature, $\delta \mathcal{V}\left(T_{c}, V\right) \sim-m^{2} T_{c}^{2} / 12$ and, using the approximation (4.9) for $T_{c}$, we see that $\left|\delta \mathcal{V}\left(T_{c}, V\right)\right|=\mathcal{O}\left(m^{3} V\right) \ll|\mathcal{V}(V)|$, implying that the estimate (4.9) is valid to leading order in $m / V$ and that in the same approximation $\chi_{T_{c}}=V$.

We infer that the electroweak phase transition is likely to have been first-order, at least in the limit of small dilaton mass $m$, as might have been expected from the 'ColemanWeinberg' form of the effective dilaton potential $\mathcal{V}(\chi)$ (see also $[87,88]) .{ }^{14}$

\subsection{Tunnelling and supercooling}

Since the phase transition is first-order, it will have proceeded by tunnelling, and it is to be expected that the early Universe supercooled to some extent before completing the transition. The rate for tunnelling between two vacua whose energy densities differ by $\Delta \mathcal{V}$ is

$$
\Gamma \sim \Lambda \times \exp \left(-\frac{27 \pi^{2}}{2} \frac{S_{0}^{4}}{(\Delta \mathcal{V})^{3}}\right)
$$

where one expects $\Lambda \sim V$ and the bounce action $S_{0}$ is given in the thin-wall approximation by

$$
S_{0}(\text { thin }- \text { wall })=\int_{0}^{V} \sqrt{2 \mathcal{V}} d \chi \equiv \alpha \sqrt{B} V^{3} .
$$

Integrating naively the logarithmic potential (2.1) would yield $\alpha=0.37 \sqrt{2}$, but this potential is not accurate close to the origin at finite temperatures. If instead one integrates (4.11) from $V / 2$ to $V$ and doubles the result, one finds $\alpha \sim 0.26 \sqrt{2}$ : for numerical estimates we estimate $\alpha \sim 0.4$. The condition for completing the transition is $\Gamma / H \sim 1$, where $H$ is the Hubble expansion rate. This condition would be satisfied if there was a temperature $T<T_{c}$ :

$$
\ln \left(\left(\frac{3}{2 \pi B}\right)^{1 / 2} \frac{m_{P}}{V}\right) \sim \frac{27 \pi^{2}}{2} \frac{\alpha^{4} B^{2} V^{12}}{\left(\Delta \mathcal{N} \pi^{2} / 30\right)^{3}\left(T_{c}^{4}-T^{4}\right)^{3}},
$$

where $\Delta \mathcal{N}$ here is the difference between the numbers of light particles before and after tunnelling, which we estimate to be $\Delta \mathcal{N}=\mathcal{O}(100)$ as discussed previously, and we have used the Hubble parameter corresponding to the vacuum energy density. It is easy to see that the condition (4.12) cannot be satisfied for representative values of $m / V \sim 1 / 10-1$ corresponding to $B \sim 1 / 400-1 / 4$, if we use the thin-wall approximation (4.11) to evaluate the tunnelling rate $(4.10)$. Using the high-temperature form $(16 \pi / 3)\left(S_{0}^{3} /(\Delta \mathcal{V})^{2}\right)$ for the bounce action yields the same conclusion, so we infer that the transition must occur at some temperature $T \ll T_{c}$ where the barrier is very low and the thin-wall approximation (4.11) is inapplicable $[87,88] .{ }^{15}$

Therefore, we explore the possibility that the supercooled transition takes place at a nucleation temperature $T_{n}=\epsilon T_{c} \ll T_{c}$ to a 'small' value $\chi_{\epsilon} \ll V$ of the dilaton field where

$$
\mathcal{V}\left(\chi_{\epsilon}\right) \sim-\mathcal{N} T_{n}^{4}=-\mathcal{N} \epsilon^{4} T_{c}^{4}=-\epsilon^{4} \frac{B V^{4}}{4}
$$

\footnotetext{
${ }^{14} \mathrm{~A}$ priori, this re-opens the possibility of electroweak baryogenesis, a possibility excluded in the Standard Model by the LEP lower limit on $m_{H}$. However, realizing this option in practice would require additional $\mathrm{CP}$ violation beyond the Kobayashi-Maskawa phase, an issue beyond the scope of this work.

${ }^{15}$ For similar conclusions in analogous models see [90, 91].
} 


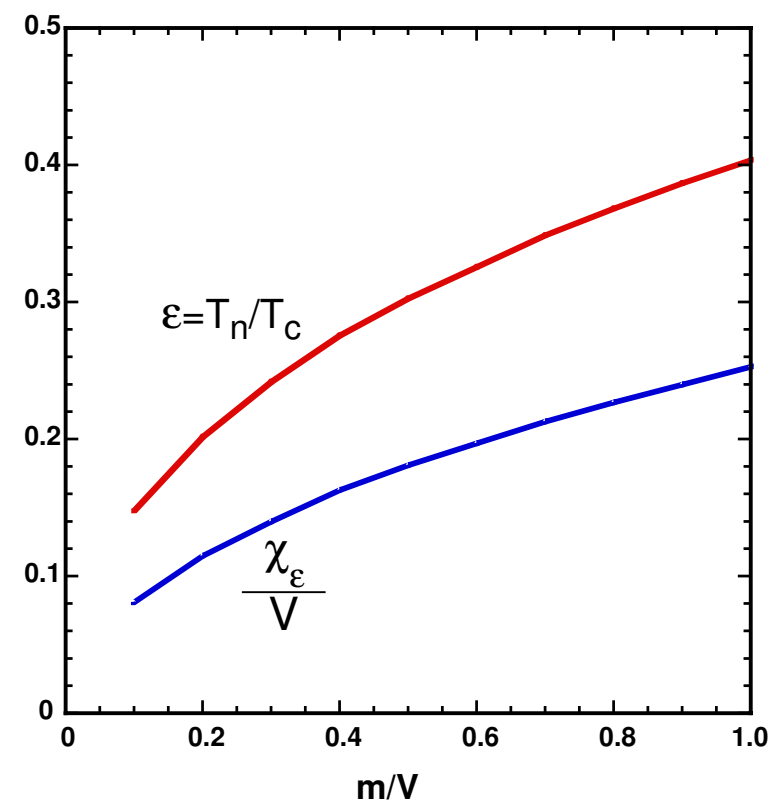

Figure 2. The ratio of the nucleation temperature $T_{n}$ relative to the critical temperature $T_{c}$, $\epsilon \equiv T_{n} / T_{c}$, and the ratio of the corresponding value of $\chi$ at nucleation, $\chi_{\epsilon} \equiv \chi\left(T_{n}\right)$, to the zerotemperature v.e.v. of the pseudo-dilaton field $V \equiv\langle 0|\chi| 0\rangle$, as functions of the ratio of the dilaton mass $m$ to the zero-temperature v.e.v. of $\chi, V$.

which reduces to solving the condition

$$
\left(\frac{\chi_{\epsilon}}{V}\right)^{4}\left[\ln \left(\chi_{\epsilon} / V\right)-\frac{1}{4}\right]=-\frac{\epsilon^{4}}{4} .
$$

Corresponding to $\chi_{\epsilon}$, one may estimate $\alpha_{\epsilon} \equiv \int_{0}^{\chi_{\epsilon}} \sqrt{2 \mathcal{V}} d \chi /\left(\sqrt{B} V^{3}\right)$, and then solve the $\Gamma / H \sim 1$ consistency condition, which may be cast in the form

$$
\epsilon=\left[1-\left(\frac{864 \pi^{2} \alpha_{\epsilon}^{4}}{B \ln \left((3 / 2 \pi B)^{1 / 2} m_{P} / V\right)}\right)^{1 / 3}\right]^{1 / 4} .
$$

Using this procedure, for $B \sim 1 / 400$, corresponding to $m / V=1 / 10$, we estimate

$$
\epsilon=\frac{T_{n}}{T_{c}}=0.15 ; \frac{\chi_{\epsilon}}{V}=0.08 ; \alpha_{\epsilon}=0.04 \sqrt{2} ;
$$

i.e., the Universe supercools substantially to a temperature $\sim 0.15$ of $T_{c}$ before completing a first-order electroweak phase transition to a dilaton v.e.v. $\sim 0.08$ of its present value $V$. Figure 2 illustrates the $m$ dependences of $\epsilon \equiv T_{n} / T_{c}$ and $\chi_{\epsilon} / V$, where $\chi_{\epsilon} \equiv \chi_{T_{n}}$. We see that $T_{n} \ll T_{c}$, and that also the value of $\chi$ at nucleation $\chi_{\epsilon} \ll V$.

In making the above estimate, we have assumed that the Universe is radiationdominated for temperatures $T>T_{c}$. Both before and after nucleation, the scalar field energy density is given approximately by $\mathcal{V}(0)=B V^{4} / 4$, and radiation in fact dominates 
the overall energy density at temperatures $T>T_{e q}$, where

$$
T_{e q} \simeq\left(\frac{30 \mathcal{V}(0)}{\pi^{2} \mathcal{N}}\right)^{1 / 4}=\left(\frac{30}{\pi^{2} \mathcal{N}}\right)^{1 / 4}\left(\frac{B}{4}\right)^{1 / 4} \simeq 0.21 \sqrt{m V} .
$$

The estimate (4.17) of $T_{e q}$ is somewhat smaller than the estimate (4.9) of the critical temperature $T_{c}$, but rather larger than the estimate (4.16) of the nucleation temperature $T_{n}$. This indicates that, for some period around the time of nucleation, the scalar field energy density dominates somewhat over radiation. However, the dilaton field rolls very quickly, with a characteristic time scale $t_{\text {roll }} \sim 200 / V$, which is very short compared to the nucleation time scale $t_{n} \sim 10^{18} / \mathrm{V}$, and decays with a characteristic rate that is very large compared with the Hubble expansion rate. Hence the Universe does not enter a de Sitter expansion phase (inflationary epoch), because the conventional slow-roll conditions

$$
\frac{1}{16 \pi G_{N}}\left(\frac{\mathcal{V}^{\prime}}{\mathcal{V}}\right)^{2} \ll 1, \frac{1}{8 \pi G_{N}}\left(\frac{\mathcal{V}^{\prime \prime}}{\mathcal{V}}\right) \ll 1
$$

where $G_{N}$ is Newton's constant, are strongly violated.

The above discussion is summarized in figure 3, in which the solid blue (red) lines represent the evolution of the cosmological energy density, $\rho$, for $m / V=1(0.1)$. Relativistic particles, including electroweak constituents (which we estimate to contribute $\Delta \mathcal{N} \simeq 100$ ) as well as Standard Model particles (which contribute $\Delta \mathcal{N}=427 / 4$ ) dominate $\rho$ until it falls to the temperature $T_{e q}$ at which it becomes equal to the scalar field energy, which $\simeq \mathcal{V}(0)$. Following the change in the rate of evolution of $\rho$, the scalar field energy density dominates $\rho$ during a short epoch that is terminated by nucleation and (almost immediately) percolation of bubbles of the low-energy electroweak vacuum. Since the dilaton field rolls and decays with a rate $\propto \alpha_{\chi} m$ where $\alpha_{\chi}$ is some coupling strength, that is much larger than the Hubble expansion rate, the scalar field energy density is then converted rapidly and efficiently to relativistic Standard Model particles, as indicated by the later kinks in the solid lines. The dotted lines represent the evolution of the energy density of relativistic particles during the short epoch of scalar field energy domination.

The short period of non-adiabatic expansion between $T_{e q}$ and $T_{n}$ is accompanied by a corresponding growth in entropy. The growth in the scale factor is by a factor $\simeq R_{n} / R_{e q}=$ $\exp \left[H\left(t_{n}-t_{e q}\right)\right]=T_{e q} / T_{n} \sim 1.9 / \sqrt{m[\mathrm{TeV}]}$ for $1 \mathrm{TeV}>m>100 \mathrm{GeV}$, leading to a corresponding growth in the entropy by a factor of $\left(R_{n} / R_{e q}\right)^{3} \sim 7$ to 200 . This is not a big problem for baryogenesis before the electroweak phase transition, but this entropy growth should be taken into account, particularly if the dilaton mass $m \sim 100 \mathrm{GeV}$.

\subsection{Percolation}

The transition to the new electroweak vacuum would have been completed at the percolation time $t_{p}$, when the Universe was filled with bubbles of true vacuum [89]. In order to estimate this, we first recall that the probability for bubble nucleation is

$$
\mathcal{P} \sim V^{4} e^{-\mathcal{B}}
$$




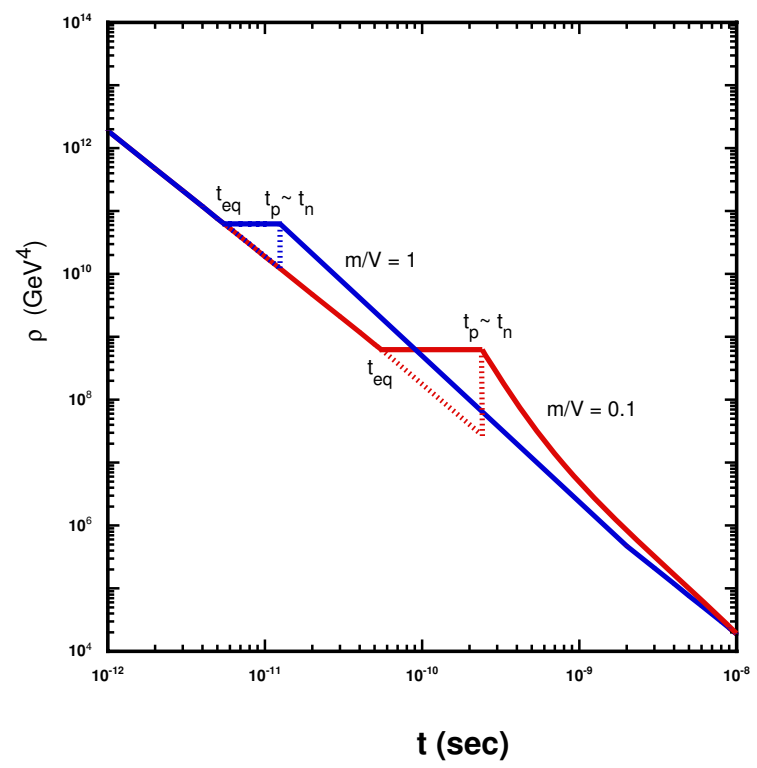

Figure 3. Illustration of the cosmological evolution of the energy density, $\rho$, for $m / V=1$ and 0.1 (blue and red solid lines, respectively). At high temperatures $T>T_{e q}, \rho$ is dominated by relativistic particles, including electroweak constituents as well as Standard Model particles. This is followed by a short epoch during which $\rho$ is dominated by the scalar field energy and the energy density of relativistic particles drops (dotted lines), which is terminated by nucleation and percolation of bubbles of the electroweak vacuum. The scalar field energy density is then converted rapidly to relativistic Standard Model particles.

where the bounce action

$$
\mathcal{B} \sim \frac{27 \pi^{2}}{2} \frac{\alpha^{4} B^{2} V^{12}}{\mathcal{N}^{3} T_{c}^{12}}
$$

where $\mathcal{N} T_{c}^{4}=B V^{4} / 4$. Following nucleation, the fractional volume of space remaining in the false vacuum is

$$
f(t) \sim \exp \left[-\int_{t_{n}}^{t} d \tau \mathcal{P}(\tau) a(\tau)^{3} V(\tau, t)\right]
$$

where

$$
V(\tau, t)=\frac{4 \pi}{3}\left(\int_{\tau}^{t} \frac{d \tau^{\prime}}{a\left(\tau^{\prime}\right)}\right)^{3}
$$

For definiteness, we assume vacuum domination, so that $a(t) \sim e^{H t}$. We find that the percolation time $t_{p}=t_{n}(1+\delta)$, where the small correction $\delta$ is found by solving the equation

$$
-\ln f=\frac{\pi}{3} t_{n}^{4} \mathcal{P} \delta^{4}
$$

which yields the estimate

$$
\delta \sim \frac{10^{4}}{V t_{n}} \sim \frac{10^{4} T_{n}^{2}}{V M_{P}} \ll 1 .
$$

We conclude that the phase transition would have completed almost immediately after nucleation, with the percolation time $t_{p} \sim t_{n}$. This conclusion is independent of the exact expansion rate between $t_{n}$ and $t_{p}$. 


\subsection{Confinement}

As was pointed out in $[47,48]$, it is a natural possibility to identify the high-temperature dilaton phase transition with the deconfinement transition. In the case of QCD, the argument was as follows. In the nonlinear Lagrangian for pseudoscalar mesons with no dilaton, the Skyrmion mass $\propto f_{\pi}$, and the Skyrmion radius $\propto 1 / f_{\pi}$. Interpreting the Skyrmion as a baryon, we interpret the low-mass, large-radius limit as $f_{\pi} \rightarrow 0$ as the quark deconfinement limit. Finally, since $m_{\pi}^{2} f_{\pi}^{2}=m_{q}\langle 0|\bar{q} q| 0\rangle$ in QCD, the chiral transition when $\langle 0|\bar{q} q| 0\rangle \rightarrow 0$ requires the vanishing of $f_{\pi}$ and hence deconfinement in QCD.

The next question is the relationship of the chiral condensate $\langle 0|\bar{q} q| 0\rangle \rightarrow 0$ to the dilaton v.e.v. In the nonlinear QCD Lagrangian with a dilaton, $V=\langle 0|\chi| 0\rangle \neq 0$ is in fact required in order that $\langle 0|\bar{q} q| 0\rangle \neq 0[47,48]$. The explicit construction of a Skyrmion solution to this theory demonstrated that the baryon mass $\rightarrow 0$ and the baryon radius $\rightarrow \infty$ when $V \rightarrow 0$. If $\langle 0|\bar{q} q| 0\rangle$ vanishes at the same temperature as the dilaton v.e.v., $V$, the chiral symmetry/deconfinement transition is identified with spontaneous scale symmetry breaking.

On the other hand, as also pointed out in $[47,48]$, it is possible that the chiral finitetemperature corrections to the effective chiral Lagrangian, cf (4.1), drive $\langle 0|\bar{q} q| 0\rangle \rightarrow 0$ even if $V=\langle 0|\chi| 0\rangle \neq 0$. In this case the chiral symmetry/confinement transition would be distinct from the dilaton transition, taking place at a lower temperature. In the case of QCD with two (and one moderately) light flavours, it was not possible on the basis of the effective low-energy theory alone to determine whether finite-temperature chiral corrections drive $\langle 0|\bar{q} q| 0\rangle$ and $f_{\pi} \rightarrow 0$ below the dilaton transition temperature, or not. However, lattice calculations of QCD seem to favour strong simultaneous variations in the $\bar{q} q$ and gluon condensates around the quark-gluon/hadron transition, consistent with the coincidence of chiral symmetry breaking, quark confinement and the development of a dilaton v.e.v.

In the electroweak case, by analogy, the formation of a dilaton v.e.v. $V \neq 0$ is necessary for the breaking of chiral symmetry that leads to the nonlinear electroweak effective Lagrangian. Moreover, on the basis of the Skyrmion model for electroweak baryons we argue that the breaking of electroweak chiral symmetry can be identified with pseudoconfinement, and expect that the electroweak baryon $\mathcal{B}$ has a mass $m_{\mathcal{B}}=\mathcal{O}(V)$, with a numerical coefficient that depends on the underlying strongly-interacting theory.

The remaining question is whether the chiral transition coincides with the dilaton transition, or whether it occurs at a lower temperature, driven by the finite-temperature corrections (4.1). On the basis of this formula, and more detailed evaluations of higherorder finite-temperature corrections in the QCD case $[85,86]$, it seems reasonable to hypothesize that they would not drive $v \rightarrow 0$ at a temperature below $\sim 2 v \sim 500 \mathrm{GeV}$. On the other hand, (4.9) suggests that $T_{c} \sim 100 \mathrm{GeV}$, and we saw subsequently that there is likely to have been supercooling by a factor $\sim 10$ before the transition to $V \neq 0$. According to the above estimates, the chiral finite-temperature corrections would not drive $v \rightarrow 0$ below this temperature, so we expect that the transitions to $V \neq 0$ and $v \neq 0$ occurred simultaneously, and we identify this transition with the pseudo-confinement of whatever underlying constituents there may have been. 


\subsection{Electroweak baryon-to-entropy ratio}

The above discussion of electroweak baryons provides a framework for re-examining the longstanding suggestion that technibaryons might provide the astrophysical cold dark matter [54]. ${ }^{16}$ When the electroweak phase transition was completed at the time of percolation by bubbles filling space, the relative orientations of the chiral condensates in the bubble interiors would have been partially mismatched, in general, leading à la Kibble to the appearance at their boundaries of topologically stable defects [93]. The number density of the defects at the time of percolation would have been of the same order as the bubble density at percolation, ${ }^{17}$ as there is a natural topological duality between the bubbles and the defect sites. These topologically-stable defects are the Skyrmions of the chiral theory, which we interpret as the electroweak baryons of the underlying strongly-coupled electroweak symmetry-breaking sector, for the reasons presented earlier.

By the time of percolation, the Universe would have filled with bubbles of the new vacuum with a characteristic size $R$ given by

$$
R \simeq \frac{3 S_{0}}{\Delta \mathcal{V}}
$$

where $S_{0}=\alpha_{\epsilon} \sqrt{B} V^{3}$ is the bubble action (4.11), and $\Delta \mathcal{V} \simeq \mathcal{N} T_{c}^{4}$. Using the estimate (4.16) for $\alpha_{\epsilon}$, we find

$$
R \simeq \frac{12 \alpha_{\epsilon}}{\sqrt{B} V} \simeq 24 \frac{\alpha_{\epsilon}}{m} \simeq \frac{1.35}{m} .
$$

It is clear that $R \ll H^{-1} \simeq(3 / 2 \pi B)^{1 / 2}\left(M_{P} / V^{2}\right) \simeq(6 / \pi)^{1 / 2}\left(M_{P} / m V\right)$.

If there is one electroweak baryon $\mathcal{B}$ per bubble, we may estimate their density to be

$$
n_{\mathcal{B}} \sim \frac{1}{R^{3}} \sim 0.4 m^{3},
$$

which can be compared with the entropy density

$$
s \sim \rho^{3 / 4} \sim H^{3 / 2} M_{P}^{3 / 2} \sim \frac{m^{3 / 2} V^{3 / 2}}{8},
$$

yielding an electroweak baryon-to-entropy ratio

$$
\frac{n_{\mathcal{B}}}{s} \sim 3.2\left(\frac{m}{V}\right)^{3 / 2}
$$

This estimate is indisputably approximate, but it does suggest strongly that the baryonto-entropy ratio is likely to be large immediately after percolation.

However, this prediction of the Kibble-like mechanism for the production of electroweak baryons would have been modified if equilibrium was restored after the transition. We

\footnotetext{
${ }^{16}$ For the record, we note that electroweak baryons are expected to be sufficiently stable to serve as dark matter particles [92].

${ }^{17}$ Since, as argued earlier, we expect the electroweak phase transition in the class of models studied here to have been first-order, we do not expect modifications of the Kibble estimate of the types discussed in, e.g., $[94,95]$ to be important for our analysis.
} 
now argue that this would have been the case following reheating after the electroweak transition, and that this would have led to a much lower density of electroweak baryons.

The pseudo-dilaton would have decayed at percolation, and the residual vacuum energy density thereby released would have led to reheating. Since the pseudo-dilaton decay rate, which is $\alpha \alpha_{\chi} m$ where $\alpha_{\chi}$ is some coupling strength, is much larger than the Hubble rate after the transition, which is $\propto\left(m / M_{P}\right) m$, we expect that the reheating temperature, $T_{R}$, would have been given by

$$
\frac{\pi^{2}}{30} \mathcal{N} T_{R}^{4} \simeq \frac{1}{4} B V^{4}
$$

where $\mathcal{N} \sim \mathcal{O}(100)$ is the effective number of relativistic degrees of freedom after the transition, implying that

$$
T_{R} \simeq 0.2 \sqrt{m V} .
$$

Equilibrium would have been established following reheating if the annihilation rate of electroweak baryons was at least as large as the Hubble expansion rate, i.e., if

$$
\sigma v n \gtrsim H \simeq \sqrt{\frac{8 \pi^{3} \mathcal{N}}{90}} \frac{T^{2}}{M_{P l}}
$$

where the annihilation cross section is related to the pseudo-dilaton mass: $\sigma v \simeq 1 / 4 B\langle\chi\rangle^{2}$, $\langle\chi\rangle$ is its expectation value at reheating, and $n=\left(m_{\mathcal{B}} T / 2 \pi\right)^{3 / 2} e^{-\left(m_{\mathcal{B}} / T\right)}$. One expects that the effective electroweak baryon mass $m_{\mathcal{B}} \propto\langle\chi\rangle$.

Thus equilibrium would have been established if:

$$
\frac{1}{4 B\langle\chi\rangle^{2}}\left(\frac{m_{\mathcal{B}}}{2 \pi T}\right)^{3 / 2} T e^{-\left(m_{\mathcal{B}} / T\right)} \gtrsim \frac{\sqrt{8 \pi^{3} \mathcal{N} / 90}}{M_{P}} .
$$

Defining $x \equiv\left(m_{\mathcal{B}} / T\right)$, this condition becomes

$$
\left(32 \pi^{6} \mathcal{N} / 45\right)^{1 / 2} \frac{e^{x}}{\sqrt{x}} \lesssim \frac{M_{P} m_{\mathcal{B}}}{m^{2}},
$$

which may usefully be written in the form $x \gtrsim x_{F}$, where the freeze-out temperature $x_{F}$ may be expressed in the form

$$
x_{F}=\ln \left(\frac{M_{P}}{V}\right)+2 \ln \left(\frac{V}{m}\right)+\ln \left(\frac{m_{\mathcal{B}}}{V}\right)-\frac{1}{2} \ln \left(32 \pi^{6} \mathcal{N} / 45\right)+\frac{1}{2} \ln \left(x_{F}\right) .
$$

We find the approximate value $x_{F} \simeq 40$, which is relatively insensitive to variations in $V / m \in(1,10)$ and even less so to $m_{\mathcal{B}} / V$ if it is $\mathcal{O}(1)$, as expected, corresponding to a freeze-out temperature

$$
T_{F} \simeq \frac{m_{\mathcal{B}}}{40} .
$$

Comparing with the estimate (4.31) of the reheating temperature $T_{R}$, we see that $T_{R}>T_{F}$, and hence that equilibrium is expected to have been achieved for $m / V \gtrsim 0.015$ and $m_{\mathcal{B}} \simeq V$. 
Once brought into equilibrium, the freeze-out density of electroweak baryons is given simply by $n\left(T_{F}\right)$, namely

$$
\frac{n_{\mathcal{B}}}{n_{\gamma}} \simeq\left(\frac{m_{\mathcal{B}}}{2 \pi T_{F}}\right)^{3 / 2} e^{-\left(m_{\mathcal{B}} / T_{F}\right)} .
$$

Using the estimate $T_{F}=m_{\mathcal{B}} / 40$ gives

$$
\eta_{\mathcal{B}}=\frac{n_{\mathcal{B}}}{n_{\gamma}} \simeq 10^{-16}
$$

which is far below the Kibble estimate (4.29) and in general agreement with the estimate in [53]. A more detailed calculation would be required to verify how accurately (4.38) is satisfied, but it is at least a useful lower limit.

For comparison, requiring the electroweak baryon density today to be $\sim 5$ times the baryon density [96], taking the baryon-to-photon ratio $\eta \sim 6 \times 10^{-10}$ and assuming a nominal electroweak pseudo-baryon mass $\sim 1 \mathrm{TeV}$, we estimate the electroweak baryon-to-photon ratio to be

$$
\eta_{\mathcal{B}} \sim 3 \times 10^{-12}
$$

The estimate (4.38) based on the freeze-out abundance is much smaller, suggesting that some mechanism for creating an electroweak pseudo-baryon asymmetry is needed if the electroweak pseudo-baryons are to provide all the dark matter [54]. However, we do not enter here into the details of any possible mechanism for generating such a electroweak pseudo-baryon asymmetry. Also, we note the possibility that incomplete equilibration might have left the baryon density in the interesting range (4.39), in which case an electroweak pseudo-baryon asymmetry might be unnecessary.

\section{Electroweak baryons as dark matter}

In this section, we assume there is a cosmological electroweak baryon asymmetry of the appropriate magnitude for the the electroweak baryons to acquire the appropriate relic density $\Omega_{\mathcal{B}} h^{2} \sim 0.1$ [96]. Since they interact strongly at the TeV scale, one might wonder whether their self-interactions would modify the standard WIMP scenario for dark matter. The self-interactions would be dominated by exchanges of the lightest available particles in the spectrum, namely the pseudo-dilaton and the massive electroweak gauge bosons. These yield self-interacting cross sections that are $\mathcal{O}(1) /(100 \mathrm{GeV})^{2}$ at most, an upper bound strong enough for their self-interactions to have no impact on the evolution of structures in the Universe.

We now study in more detail the direct interactions of electroweak baryons with conventional matter, which would also be dominated by exchanges of the pseudo-dilaton and the massive electroweak gauge bosons $W^{ \pm}$and $Z^{0}$. At this stage, we must distinguish the generic possibilities of bosonic and fermionic electroweak baryons. As discussed earlier, if the lowest-lying electroweak baryon state is bosonic, it is expected to have $I=J=0$, whereas if it is fermionic it is expected to have $I=J=1 / 2$, like the conventional nu- 
cleons. ${ }^{18}$ In the bosonic case, therefore, one need only consider pseudo-dilaton, $\gamma$ and $Z^{0}$ exchanges, whereas $W^{ \pm}$exchange should also be considered in the fermionic case.

The questions also arise in the latter case which of the two (nearly) degenerate states is lighter, and what is its electric charge. The answer to the latter question should clearly be that the ground state is neutral, or the standard WIMP paradigm would fail. ${ }^{19}$ As discussed in [98], two classes of diagrams are likely to contribute to the mass differences between the $I_{3}= \pm 1 / 2$ fermionic partners: (a) electromagnetic 'self-energy' diagrams and (b) photon-exchange 'Coulomb potential' diagrams. We expect these diagrams to have the following orders of magnitude:

$$
\mathcal{O}\left(\frac{\alpha}{4 \pi}\right) V \times\left[(\mathrm{a}) \Sigma_{i} q_{i}^{2} \text { or }\left(\Sigma_{i} q_{i}\right)^{2},(\mathrm{~b}) \Sigma_{i \neq j} q_{i} q_{j}\right] .
$$

It is plausible that, for any odd number of fermionic constituents of electroweak baryons, there are charge assignments that ensure that the lighter of the two $I=1 / 2$ states is electrically neutral. In the specific case of QCD, the fact that the neutron is heavier than the proton is ascribed to the fact that $m_{d}>m_{u}$, whereas the electromagnetic mass difference alone is calculated to yield $m_{p}>m_{n}$, so QCD actually supports the plausibility of this expectation, despite the neutron being heavier! In any such scenario, the mass difference with the heavier member of the $I=1 / 2$ doublet, as given by (5.1), is expected to be $\mathcal{O}(1) \mathrm{GeV}$ or more, as would also be given by scaling up the electromagnetic contribution to the $p-n$ mass difference. We therefore expect that the heavier (charged) electroweak baryon would $\beta$-decay with a lifetime $\mathcal{O}\left(10^{-11}\right)$ s or less.

The electroweak baryon asymmetry might have either sign, so that the residual excess of electroweak baryons might have electromagnetic charges $(0,+1)$ or $(-1,0)$. Metastable charge -1 particles could affect Big Bang nucleosynthesis via catalysis reactions that are absent for metastable charge +1 particles [99]. However, the above estimate indicates that any charged electroweak baryons would decay before Big Bang nucleosynthesis, so it is unnecessary to consider the corresponding constraints. Henceforward, we use the notation $(\mathcal{N}, \mathcal{P})$ that is natural for an $(0,+1)$ doublet, but our remarks apply equally to the $(-1,0)$ case.

In general, the rate for weak-interaction $\mathcal{P} \leftrightarrow \mathcal{N}$ transitions at finite temperature is estimated to be

$$
\Gamma_{\mathcal{P} \leftrightarrow \mathcal{N}} \sim G_{F}^{2} T^{5},
$$

which may be compared with the Hubble expansion rate $H \sim \sqrt{g_{*} G_{N}} T^{2}$, where $g_{*}$ is the effective number of relativistic degrees of freedom, which are assumed to dominate the cosmological expansion. This comparison suggests that the weak interactions of the electroweak baryons freeze out at

$$
T_{F} \sim\left(\frac{g_{*} G_{N}}{G_{F}^{4}}\right)^{1 / 6} \sim 1 \mathrm{MeV},
$$

\footnotetext{
${ }^{18} \mathrm{We}$ also note that, in the case of an $\mathrm{Sp}(\mathrm{N})$ gauge group, there would be no stable electroweak baryons, as the electroweak Skyrmions would decay into bosons.

${ }^{19}$ For a discussion in the context of 'little Higgs' models see [97].
} 
just like the weak interactions of conventional baryons. This would suggest a relic abundance of $\mathcal{P}$ relative to $\mathcal{N}$ :

$$
\frac{n_{\mathcal{P}}}{n_{\mathcal{N}}} \sim e^{-\Delta M / T_{F}} \sim e^{-10^{3}}
$$

which is completely negligible.

However, this argument neglects the possible formation of charged pseudo-nuclei that might be $\beta$-stable, and be capable of trapping the otherwise-unstable $\mathcal{P}$ electroweak baryons. Any substantial relic abundance of such objects would be incompatible with upper limits on possible charged relics from the Big Bang. In the case of conventional Big-Bang nucleosynthesis, the formation of light nuclei is inhibited by photo-dissociation until the density of photons above the nuclear photo-dissociation threshold falls below the density of baryons, which occurs when the temperature $T \sim 0.1 \mathrm{MeV}$, below the freeze-out temperature (5.3). In our electroweak baryon case, the density of photons above the photopseudo-dissociation threshold $\sim 2 \mathrm{GeV}$ falls below that of the electroweak baryons when

$$
e^{-2 \mathrm{GeV} / T} \sim \eta_{\mathcal{B}}
$$

where $\eta_{\mathcal{B}}$ is given in (4.39). This condition is reached when the temperature falls to $T \sim 75 \mathrm{MeV}$, at which epoch

$$
\frac{n_{\mathcal{P}}}{n_{\mathcal{N}}} \sim e^{-\Delta M / T} \sim 10^{-7}
$$

assuming $m_{\mathcal{P}}-m_{\mathcal{N}} \sim 10^{3}\left(m_{n}-m_{p}\right)$. We conclude that the relative abundance of charged electroweak baryons $\mathcal{P}$ is suppressed strongly below that of neutral electroweak baryons $\mathcal{N}$ well before the formation of pseudo-nuclei begins. However, the suppression (5.6) translates into an abundance relative to protons of

$$
\frac{n_{\mathcal{P}}}{n_{p}} \sim 10^{-10}
$$

which may not be sufficient by itself to respect the strong upper limits on the possible abundance of charged relics from the Big Bang. Note that a even a slightly larger mass difference between $\mathcal{P}$ and $\mathcal{N}$, will greatly suppress the abundance of $\mathcal{P}$ to $p$.

Detailed exploration of this question requires deeper studies of pseudo-nuclear physics and Big-Bang pseudo-nucleosynthesis that go beyond the scope of this paper. However, we note that if the abundance of charged relics turns out to be problematic, there are at least two escape routes. One is that the electroweak baryons are bosons, in which case the lowest-lying state has $I=J=0$, and the other is that the underlying gauge group is of $\mathrm{Sp}(\mathrm{N})$ type, in which case electroweak baryons are unstable.

\section{Detection of electroweak baryonic dark matter}

The scattering of electroweak baryonic dark matter on conventional matter is expected to be dominated by exchanges of the photon, the neutral electroweak gauge boson $Z^{0}$ and the pseudo-dilaton $\chi \cdot^{20}$

\footnotetext{
${ }^{20}$ Scattering of a fermionic electroweak baryon via $W^{ \pm}$exchange would entail inelastic charge-exchange: $\mathcal{N} p \rightarrow \mathcal{P} n$. Since this requires an excitation energy $\mathcal{O}(\mathrm{GeV})$, far exceeding the expected kinetic energy of the relic dark matter particle, we neglect this contribution.
} 
The photon-exchange contribution to technibaryon scattering on conventional matter was first discussed in [55]. It was pointed out there that the electromagnetic scattering of scalar electroweak baryons would proceed via their charge radius, which is quite model-dependent. It was estimated that the rate would be relatively small (see also [100]), and quite possibly below the reach of current experiments. On the other hand, it was also pointed out in [55] that fermionic electroweak baryons would scatter via their magnetic-dipole moments, yielding considerably larger rates, of order 1 event $/ \mathrm{kg} /$ day. A rate as large as this has now been excluded by experiment [101], suggesting that fermionic electroweak baryons could not constitute the astrophysical dark matter.

Turning to $Z^{0}$ exchange, it would contribute to the scattering of a fermionic electroweak baryon via vertices that are both spin-independent and-dependent, but we do not discuss these in detail for the reason given in the previous paragraph. On the other hand, $Z^{0}$-exchange would not contribute to the scattering of a bosonic electroweak baryon, because it has $I=Y=0$.

It remains to consider the pseudo-dilaton exchange contribution to the scattering of bosonic electroweak baryons $[100,102]$. Experiments usually quote upper limits on the cross section on an individual nucleon $n$, neglecting the possible difference between the proton and neutron. Neglecting the photon exchange contribution, the cross section may be written in the form [100]

$$
\sigma_{\mathcal{B}}=\frac{m_{R}^{2}}{4 \pi}\left[\frac{f m_{n} M_{\mathcal{B}}}{V^{2} m^{2}}\right]^{2}
$$

where $m_{R}=M_{\mathcal{B}} m_{n} /\left(M_{\mathcal{B}}+m_{n}\right)$ is the reduced electroweak baryon mass. In writing (6.1) we assume that all the electroweak baryon mass is due to the pseudo-dilaton v.e.v. $V$, and the coefficient $f$ is defined and calculated as follows.

Following [103, 104], we have

$$
f=\sum_{q=u, d, s} f_{q}+\frac{6}{27} f_{G},
$$

where for the light quarks $q=u, d, s$, we have:

$$
\begin{aligned}
& f_{u}=\frac{m_{u} B_{u}}{m_{N}}=\frac{2 \Sigma_{\pi N}}{m_{N}\left(1+\frac{m_{d}}{m_{u}}\right)\left(1+\frac{B_{d}}{B_{u}}\right)} \\
& f_{d}=\frac{m_{d} B_{d}}{m_{N}}=\frac{2 \Sigma_{\pi N}}{m_{N}\left(1+\frac{m_{u}}{m_{d}}\right)\left(1+\frac{B_{u}}{B_{d}}\right)} \\
& f_{s}=\frac{m_{s} B_{s}}{m_{N}}=\frac{\left(\frac{m_{s}}{m_{d}}\right) \Sigma_{\pi N} y}{m_{N}\left(1+\frac{m_{u}}{m_{d}}\right)}
\end{aligned}
$$

and $f_{G}=1-\sum_{q=u, d, s} f_{q}$, where $\Sigma_{\pi N}$ is the $\pi$-nucleon $\sigma$-term, $m_{q} B_{q}=\left\langle n\left|m_{q} \bar{q} q\right| n\right\rangle$, $y=1-\sigma_{0} / \Sigma_{\pi N}$, and octet baryon mass differences give $\sigma_{0}=36 \pm 7 \mathrm{MeV}$. Inserting the following representative numerical values: $\Sigma_{\pi N}=50 \mathrm{MeV}, m_{d} / m_{u}=1.81, B_{d} / B_{u}=0.75$ and $m_{s} / m_{d}=18.9$, we estimate

$$
f_{u}=0.022, f_{d}=0.029, \quad f_{s}=0.182, f_{G}=0.767,
$$




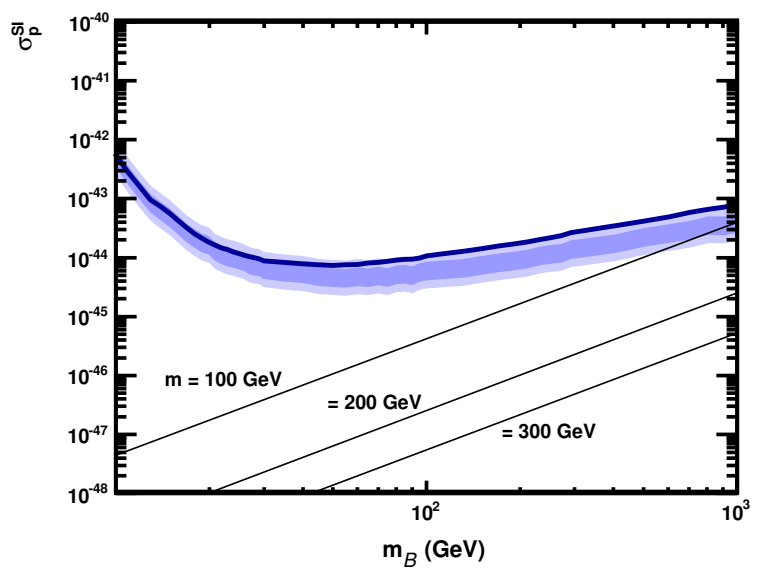

Figure 4. The estimated cross section for the elastic scattering of a scalar electroweak boson $\mathcal{B}$ on a proton, as a function of $m_{\mathcal{B}}$, assuming $V=1 \mathrm{TeV}$ and the pseudo-dilaton mass $m=100,200$ and $300 \mathrm{GeV}$. See the text for a discussion of the uncertainties in hadronic inputs to this calculation. Also shown is the published upper limit of the XENON100 experiment for dark matter particles masses $\leq 1 \mathrm{TeV}$ (solid blue line) and its expected sensitivity (blue band) [101].

and hence $f \simeq 0.40$, though with considerable uncertainty, related in particular to the poorly-determined experimental value of $\Sigma_{\pi N}$. Varying this between 36 and $72 \mathrm{MeV}$ yields over an order of magnitude range in $\sigma_{\mathcal{B}}$ for fixed values of the other parameters.

Numerically, assuming that the photon-exchange contribution is negligible, using $f=0.4$ and choosing a representative electroweak baryon mass $M_{\mathcal{B}}=V=1 \mathrm{TeV}$ and the minimum dilaton mass $m=100 \mathrm{GeV}$, we find a cross section $\sigma_{\mathcal{B}} \simeq 4.4 \times 10^{-44} \mathrm{~cm}^{2}$. This may be compared with the upper limit established by the XENON100 experiment on WIMP-nucleon scattering, which is $\sim 8 \times 10^{-44} \mathrm{~cm}^{2}$ for a WIMP mass of $1 \mathrm{TeV}$, the largest mass displayed in [101], as shown in figure 4. However, we note that the cross section (6.1) grows $\sim m_{\mathcal{B}}^{2}$, whereas the upper limit given in [101] grows more slowly in the mass range displayed. Extrapolating the published XENON100 result, as seen in figure 4, we estimate that the $\mathcal{B} p$ cross section may exceed the XENON100 sensitivity for $m_{\mathcal{B}} \gtrsim 2 \mathrm{TeV}$, if $V$ $=1 \mathrm{TeV}$ and $m=100 \mathrm{GeV}$. On the other hand, the calculated cross section decreases $\sim m^{-4}$ for larger pseudo-dilaton masses, as illustrated in figure 4 by the cases $m=200$ and $300 \mathrm{GeV}$. This discussion serves to emphasize, however, that there might be good prospects for detecting electroweak dark matter with forthcoming experiments.

\section{Summary}

We have analyzed in this paper phenomenological and cosmological aspects of what may be the best-defined extension of the Standard Model in the context of composite models with new, nearly-conformal strong interactions around the $\mathrm{TeV}$ scale. In this model there is a single additional light degree of freedom, in addition to the known Standard Model particles, a pseudo-dilaton. This particle would have couplings similar to those of a Standard Model Higgs boson, except for a universal overall suppression and the possibility 
that the loop-generated decays to $\gamma \gamma$ and $g g$ might be modified by additional particles with electric charges and/or QCD interactions. We have used the upper limits on Higgs production at the LHC [1-3] to constrain the pseudo-dilaton coupling suppression factor for dilaton masses $m>140 \mathrm{GeV}$, and have also discussed the constraints coming from precision electroweak data.

We have also discussed the cosmology of such a model, including the possible nature of the electroweak phase transition, which we expect to have been first-order except possibly for large $m$. This re-opens the possibility of generating the cosmological baryon asymmetry during the electroweak phase transition, a possibility we did not develop here. On the other hand, we do not expect that an enormous growth in entropy would have been engendered during the transition, so generating the cosmological baryon asymmetry (and possibly the cosmological electroweak baryon asymmetry) before the electroweak phase transition remains a possibility.

We identify electroweak baryons with the Skyrmion soliton solutions of the low-energy effective electroweak theory, much as conventional baryons may be described as Skyrmions in the low-energy effective chiral $\mathrm{SU}(2) \times \mathrm{SU}(2)$ or $\mathrm{SU}(3) \times \mathrm{SU}(3)$ description of QCD. In ignorance of the strong dynamics underlying electroweak symmetry breaking, the electroweak baryons may be a doublet of $I=J=1 / 2$ fermions or a singlet $I=J=0$ boson, or even unstable if the underlying gauge theory is based on an $\mathrm{SP}(\mathrm{N})$ group. However, there are two potential problems for the fermionic case: first, even if the charged member of the $I=J=1 / 2$ doublet is heavier than the neutral one, and hence unstable, some of its cosmological abundance might have become 'sequestered' in problematic charged stable 'electroweak nuclei'; secondly, the magnetic-moment scattering of neutral fermionic electroweak baryons is estimated to exceed the current upper limit [101]. On the other hand, this and other experiments are just at the verge of sensitive to scalar electroweak baryonic dark matter.

The LHC experiments will presumably soon tell us whether there is a Standard Model Higgs-like boson. If so, it would probably weigh less than about $130 \mathrm{GeV}$ and our hunch is that it would probably be accompanied by supersymmetric particles. On the other hand, if it does not exist, one should not assume that the correct theory of electroweak symmetry breaking is Higgsless. There may still exist a Higgs-like boson but with suppressed coupling: a 'less-Higgs' theory. In the event that a Standard Model-like Higgs boson is not discovered soon with a mass below $\sim 140 \mathrm{GeV}$, the LHC experiments should push the upper limits on a 'less-Higgs' boson down relentlessly. Such a 'less-Higgs' boson would necessarily be accompanied by massive states, needed to unitarize $W W$ scattering, and its discovery would be almost as exciting as proving that electroweak symmetry breaking is Higgsless.

Note added. While finishing this paper, three related papers have appeared. Constraints on a radion model have been considered recently in [105]. Refs. [106, 107] derive collider constraints on a model with a dilaton coupled to gluons via the full QCD $\beta$ function for light quarks. Because the light-quark contribution to the $\beta$ function is considerably larger than the top-quark contribution in the model we study in our section 2.3 and figure 1 , the lower limit derived in $[106,107]$ on their analogue of our dilaton v.e.v. $V$ is considerably stronger than in the model considered here. 


\section{Acknowledgments}

J.E. thanks Marek Karliner for discussions. The work of J.E. and K.A.O. was supported partly by the London Centre for Terauniverse Studies (LCTS), using funding from the European Research Council via the Advanced Investigator Grant 267352. The work of K.A.O. was also supported in part by DOE grant DE-FG02-94ER-40823 at the University of Minnesota. The work of B.A.C. was supported in part by a grant from the Natural Sciences and Engineering Research Council of Canada. The authors thank the CERN Theory Division for its hospitality.

Open Access. This article is distributed under the terms of the Creative Commons Attribution License which permits any use, distribution and reproduction in any medium, provided the original author(s) and source are credited.

\section{References}

[1] CMS collaboration, Combined Standard Model Higgs boson searches with up to $2.3 \mathrm{fb}^{-1}$ of pp collision data at $\sqrt{s}=7 \mathrm{TeV}$ at the LHC, PAS-HIG-11-023, CERN, Geneva Switzerland (2011).

[2] ATLAS collaboration, Combined Standard Model Higgs boson searches with up to $2.3 \mathrm{fb}^{-1}$ of pp collisions at $\sqrt{s}=7 \mathrm{TeV}$ at the LHC, ATLAS-CONF-2011-157, CERN, Geneva Switzerland (2011).

[3] ATLAS, CMS and LHC Higgs Combination Group collaborations, L. Rolandi, Higgs status and combinations, http://indico.in2p3.fr/getFile.py/access?contribId $=72$ \&sessionId $=19 \&$ resId $=0 \&$ materialId $=$ slides\&confId $=6004$.

[4] M. Veltman, Second threshold in weak interactions, Acta Phys. Polon. B 8 (1977) 475 [INSPIRE].

[5] C.E. Vayonakis, New threshold of weak interactions, preprint University of Athens, Athens Greece (1977) [INSPIRE].

[6] B.W. Lee, C. Quigg and H. Thacker, The strength of weak interactions at very high-energies and the Higgs boson mass, Phys. Rev. Lett. 38 (1977) 883 [INSPIRE].

[7] B.W. Lee, C. Quigg and H. Thacker, Weak interactions at very high-energies: the role of the Higgs boson mass, Phys. Rev. D 16 (1977) 1519 [inSPIRE].

[8] LEP Electroweak Working Group webpage, http://lepewwg.web.cern.ch/LEPEWWG/.

[9] GFitTer collaboration, M. Baak et al., Updated status of the global electroweak fit and constraints on new physics, arXiv:1107.0975 [INSPIRE].

[10] G. Altarelli and G. Isidori, Lower limit on the Higgs mass in the Standard Model: an update, Phys. Lett. B 337 (1994) 141 [INSPIRE].

[11] J. Casas, J. Espinosa and M. Quirós, Improved Higgs mass stability bound in the Standard Model and implications for supersymmetry, Phys. Lett. B 342 (1995) 171 [hep-ph/9409458] [INSPIRE].

[12] J. Ellis, J. Espinosa, G. Giudice, A. Hoecker and A. Riotto, The probable fate of the Standard Model, Phys. Lett. B 679 (2009) 369 [arXiv:0906. 0954] [INSPIRE]. 
[13] J.R. Ellis and D. Ross, A light Higgs boson would invite supersymmetry, Phys. Lett. B 506 (2001) 331 [hep-ph/0012067] [INSPIRE].

[14] C. Csáki, J. Hubisz and S.J. Lee, Radion phenomenology in realistic warped space models, Phys. Rev. D 76 (2007) 125015 [arXiv:0705.3844] [inSPIRE].

[15] W.D. Goldberger, B. Grinstein and W. Skiba, Distinguishing the Higgs boson from the dilaton at the Large Hadron Collider, Phys. Rev. Lett. 100 (2008) 111802 [arXiv:0708.1463] [INSPIRE].

[16] J. Fan, W.D. Goldberger, A. Ross and W. Skiba, Standard Model couplings and collider signatures of a light scalar, Phys. Rev. D 79 (2009) 035017 [arXiv:0803.2040] [INSPIRE].

[17] K. Yamawaki, Walking over the composites: in the spirit of Sakata, Prog. Theor. Phys. Suppl. 167 (2007) 127 [InSPIRE].

[18] K. Yamawaki, Quest for the dynamical origin of mass: an LHC perspective from Sakata, Nambu and Maskawa, Prog. Theor. Phys. Suppl. 180 (2010) 1 [arXiv:0907.5277] [INSPIRE].

[19] K. Yamawaki, Conformal Higgs, or techni-dilaton-composite Higgs near conformality, Int. J. Mod. Phys. A 25 (2010) 5128 [arXiv:1008.1834] [InSPIRE].

[20] S. Matsuzaki and K. Yamawaki, Techni-dilaton signatures at LHC, arXiv:1109.5448 [INSPIRE].

[21] R. Contino, C. Grojean, M. Moretti, F. Piccinini and R. Rattazzi, Strong double Higgs production at the LHC, JHEP 05 (2010) 089 [arXiv:1002.1011] [INSPIRE].

[22] J. Espinosa, C. Grojean and M. Muhlleitner, Composite Higgs search at the LHC, JHEP 05 (2010) 065 [arXiv:1003.3251] [INSPIRE].

[23] R. Contino, Tasi 2009 lectures: the Higgs as a composite Nambu-Goldstone boson, arXiv: 1005.4269 [INSPIRE].

[24] R. Contino, Hunting the composite Higgs, talk at the Higgs Hunting Workshop, http://indico2.lal.in2p3.fr/indico/getFile.py/access?contribId=27\&sessionId=11 \&resId=0\&materialId=slides\&confId=1507, Orsay France July 28-30 2011.

[25] S. Weinberg, Nonlinear realizations of chiral symmetry, Phys. Rev. 166 (1968) 1568 [INSPIRE].

[26] S.R. Coleman, J. Wess and B. Zumino, Structure of phenomenological Lagrangians. 1, Phys. Rev. 177 (1969) 2239 [inSPIRE].

[27] C.G. Callan Jr., S.R. Coleman, J. Wess and B. Zumino, Structure of phenomenological Lagrangians. 2, Phys. Rev. 177 (1969) 2247 [INSPIRE].

[28] A. Salam and J. Strathdee, Nonlinear realizations. 2. Conformal symmetry, Phys. Rev. 184 (1969) 1760 [INSPIRE].

[29] J.R. Ellis, Aspects of conformal symmetry and chirality, Nucl. Phys. B 22 (1970) 478 [INSPIRE].

[30] T. Appelquist and C.W. Bernard, Strongly interacting Higgs bosons, Phys. Rev. D 22 (1980) 200 [InSPIRE].

[31] B. Grinstein, Strong electroweak symmetry breaking, arXiv:1102.4009 [INSPIRE]. 
[32] J. Andersen et al., Discovering technicolor, Eur. Phys. J. Plus 126 (2011) 81 [arXiv:1104.1255] [INSPIRE].

[33] K. Yamawaki, M. Bando and K.-I. Matumoto, Scale invariant technicolor model and a technidilaton, Phys. Rev. Lett. 56 (1986) 1335 [InSPIRE].

[34] M. Bando, K.-I. Matumoto and K. Yamawaki, Technidilaton, Phys. Lett. B 178 (1986) 308 [INSPIRE].

[35] D.D. Dietrich, F. Sannino and K. Tuominen, Light composite Higgs from higher representations versus electroweak precision measurements: predictions for CERN LHC, Phys. Rev. D 72 (2005) 055001 [hep-ph/0505059] [INSPIRE].

[36] M. Hashimoto and K. Yamawaki, Techni-dilaton at conformal edge, Phys. Rev. D 83 (2011) 015008 [arXiv: 1009.5482] [INSPIRE].

[37] T. Appelquist and Y. Bai, A light dilaton in walking gauge theories, Phys. Rev. D 82 (2010) 071701 [arXiv:1006.4375] [INSPIRE].

[38] B. Grinstein and P. Uttayarat, A very light dilaton, JHEP 07 (2011) 038 [arXiv:1105.2370] [INSPIRE].

[39] A. Delgado, K. Lane and A. Martin, A light scalar in low-scale technicolor, Phys. Lett. B 696 (2011) 482 [arXiv: 1011.0745] [INSPIRE].

[40] O. Antipin, M. Mojaza and F. Sannino, Light dilaton at fixed points and ultra light scale super Yang-Mills, arXiv:1107.2932 [INSPIRE].

[41] B. Grinstein and P. Uttayarat, A very light dilaton, JHEP 07 (2011) 038 [arXiv:1105.2370] [INSPIRE].

[42] R. Crewther, Broken scale invariance in the width of a single dilaton, Phys. Lett. B 33 (1970) 305 [INSPIRE].

[43] R. Crewther, Nonperturbative evaluation of the anomalies in low-energy theorems, Phys. Rev. Lett. 28 (1972) 1421 [INSPIRE].

[44] M.S. Chanowitz and J.R. Ellis, Canonical anomalies and broken scale invariance, Phys. Lett. B 40 (1972) 397 [INSPIRE].

[45] M.S. Chanowitz and J.R. Ellis, Canonical trace anomalies, Phys. Rev. D 7 (1973) 2490 [INSPIRE].

[46] J.R. Ellis, M.K. Gaillard and D.V. Nanopoulos, A phenomenological profile of the Higgs boson, Nucl. Phys. B 106 (1976) 292 [INSPIRE].

[47] B. Campbell, J.R. Ellis and K.A. Olive, Effective Lagrangian approach to QCD phase transitions, Phys. Lett. B 235 (1990) 325 [INSPIRE].

[48] B.A. Campbell, J.R. Ellis and K.A. Olive, QCD phase transitions in an effective field theory, Nucl. Phys. B 345 (1990) 57 [inSPIRE].

[49] T. Skyrme, A nonlinear field theory, Proc. Roy. Soc. Lond. A 260 (1961) 127 [InSPIRE].

[50] T. Skyrme, A unified field theory of mesons and baryons, Nucl. Phys. 31 (1962) 556 [INSPIRE].

[51] J.M. Cline, M. Jarvinen and F. Sannino, The electroweak phase transition in nearly conformal technicolor, Phys. Rev. D 78 (2008) 075027 [arXiv:0808.1512] [INSPIRE]. 
[52] F. Sannino, Conformal dynamics for TeV physics and cosmology, Acta Phys. Polon. B 40 (2009) 3533 [arXiv: 0911.0931] [INSPIRE].

[53] R. Chivukula and T.P. Walker, Technicolor cosmology, Nucl. Phys. B 329 (1990) 445 [INSPIRE].

[54] S. Nussinov, Technocosmology: could a technibaryon excess provide a 'natural' missing mass candidate?, Phys. Lett. B 165 (1985) 55 [InSPIRE].

[55] J. Bagnasco, M. Dine and S.D. Thomas, Detecting technibaryon dark matter, Phys. Lett. B 320 (1994) 99 [hep-ph/9310290] [InSPIRE].

[56] M. Gell-Mann and M. Levy, The axial vector current in $\beta$ decay, Nuovo Cim. 16 (1960) 705 [INSPIRE].

[57] M. Gell-Mann, R. Oakes and B. Renner, Behavior of current divergences under $\mathrm{SU}(3) \times \mathrm{SU}(3)$, Phys. Rev. 175 (1968) 2195 [INSPIRE].

[58] J. Schechter, Effective Lagrangian with two color singlet gluon fields, Phys. Rev. D 21 (1980) 3393 [inSPIRE].

[59] A. Salomone, J. Schechter and T. Tudron, Properties of scalar gluonium, Phys. Rev. D 23 (1981) 1143 [InSPIRE].

[60] A.A. Migdal and M.A. Shifman, Dilaton effective Lagrangian in gluodynamics, Phys. Lett. B 114 (1982) 445 [inSPIRE].

[61] J.R. Ellis and J. Lanik, Is scalar gluonium observable?, Phys. Lett. B 150 (1985) 289 [INSPIRE].

[62] F. Gianotti et al., Physics potential and experimental challenges of the LHC luminosity upgrade, Eur. Phys. J. C 39 (2005) 293 [hep-ph/0204087] [INSPIRE].

[63] CLIC Physics Working Group collaboration, E. Accomando et al., Physics at the CLIC multi-TeV linear collider, hep-ph/0412251 [INSPIRE].

[64] M.A. Shifman, A. Vainshtein, M. Voloshin and V.I. Zakharov, Low-energy theorems for Higgs boson couplings to photons, Sov. J. Nucl. Phys. 30 (1979) 711 [Yad. Fiz. 30 (1979) 1368] [INSPIRE].

[65] R. Gastmans, S.L. Wu and T.T. Wu, Higgs decay $H \rightarrow \gamma \gamma$ through a $W$ loop: difficulty with dimensional regularization, arXiv:1108.5322 [INSPIRE].

[66] R. Gastmans, S.L. Wu and T.T. Wu, Higgs decay into two photons, revisited, arXiv:1108.5872 [INSPIRE].

[67] K. Fujikawa, B. Lee and A. Sanda, Generalized renormalizable gauge formulation of spontaneously broken gauge theories, Phys. Rev. D 6 (1972) 2923 [InSPIRE].

[68] M. Shifman, A. Vainshtein, M. Voloshin and V. Zakharov, Higgs decay into two photons through the $W$-boson loop: no decoupling in the $m_{W} \rightarrow 0$ limit, Phys. Rev. D 85 (2012) 013015 [arXiv:1109.1785] [INSPIRE].

[69] D. Huang, Y. Tang and Y.-L. Wu, Note on Higgs decay into two photons $H \rightarrow \gamma \gamma$, arXiv: 1109.4846 [INSPIRE].

[70] W.J. Marciano, C. Zhang and S. Willenbrock, Higgs decay to two photons, Phys. Rev. D 85 (2012) 013002 [arXiv:1109.5304] [INSPIRE]. 
[71] F. Jegerlehner, Comment on $H \rightarrow \gamma \gamma$ and the role of the decoupling theorem and the equivalence theorem, arXiv:1110.0869 [INSPIRE].

[72] H.-S. Shao, Y.-J. Zhang and K.-T. Chao, Higgs decay into two photons and reduction schemes in cutoff regularization, JHEP 01 (2012) 053 [arXiv:1110.6925] [INSPIRE].

[73] M.E. Peskin and T. Takeuchi, A new constraint on a strongly interacting Higgs sector, Phys. Rev. Lett. 65 (1990) 964 [INSPIRE].

[74] M.E. Peskin and T. Takeuchi, Estimation of oblique electroweak corrections, Phys. Rev. D 46 (1992) 381 [INSPIRE].

[75] G. Altarelli and R. Barbieri, Vacuum polarization effects of new physics on electroweak processes, Phys. Lett. B 253 (1991) 161 [INSPIRE].

[76] R. Foadi and F. Sannino, WW scattering in walking technicolor: no discovery scenarios at the CERN LHC and ILC, Phys. Rev. D 78 (2008) 037701 [arXiv:0801.0663] [InSPIRE].

[77] K. Haba, S. Matsuzaki and K. Yamawaki, S parameter in the holographic walking/conformal technicolor, Prog. Theor. Phys. 120 (2008) 691 [arXiv:0804.3668] [INSPIRE].

[78] R. Foadi, M. Jarvinen and F. Sannino, Unitarity in technicolor, Phys. Rev. D 79 (2009) 035010 [arXiv:0811.3719] [INSPIRE].

[79] A. Falkowski, C. Grojean, A. Kaminska, S. Pokorski and A. Weiler, If no Higgs then what?, JHEP 11 (2011) 028 [arXiv:1108.1183] [INSPIRE].

[80] M. Gillioz, A. von Manteuffel, P. Schwaller and D. Wyler, The little skyrmion: new dark matter for little Higgs models, JHEP 03 (2011) 048 [arXiv:1012.5288] [INSPIRE].

[81] E. Witten, Current algebra, baryons and quark confinement, Nucl. Phys. B 223 (1983) 433 [INSPIRE].

[82] J.R. Ellis and M. Karliner, An analysis of the angular momentum of the proton, Phys. Lett. B 213 (1988) 73 [INSPIRE].

[83] G. Dvali, G.F. Giudice, C. Gomez and A. Kehagias, UV-completion by classicalization, JHEP 08 (2011) 108 [arXiv:1010.1415] [InSPIRE].

[84] C. Grojean and R.S. Gupta, Theory and LHC phenomenology of classicalon decays, arXiv:1110.5317 [INSPIRE].

[85] J. Gasser and H. Leutwyler, Light quarks at low temperatures, Phys. Lett. B 184 (1987) 83 [INSPIRE].

[86] J. Gasser and H. Leutwyler, Thermodynamics of chiral symmetry, Phys. Lett. B 188 (1987) 477 [INSPIRE].

[87] T. Konstandin and G. Servant, Cosmological consequences of nearly conformal dynamics at the TeV scale, JCAP 12 (2011) 009 [arXiv:1104.4791] [INSPIRE].

[88] T. Konstandin and G. Servant, Natural cold baryogenesis from strongly interacting electroweak symmetry breaking, JCAP 07 (2011) 024 [arXiv: 1104.4793] [INSPIRE].

[89] A.H. Guth and E.J. Weinberg, Could the universe have recovered from a slow first order phase transition?, Nucl. Phys. B 212 (1983) 321 [INSPIRE].

[90] L. Randall and G. Servant, Gravitational waves from warped spacetime, JHEP 05 (2007) 054 [hep-ph/0607158] [INSPIRE]. 
[91] T. Konstandin, G. Nardini and M. Quirós, Gravitational backreaction effects on the holographic phase transition, Phys. Rev. D 82 (2010) 083513 [arXiv: 1007.1468] [INSPIRE].

[92] E. D'Hoker and E. Farhi, The decay of the skyrmion, Phys. Lett. B 134 (1984) 86 [INSPIRE].

[93] T. Kibble, Topology of cosmic domains and strings, J. Phys. A 9 (1976) 1387 [InSPIRE].

[94] W. Zurek, Cosmological experiments in superfluid helium?, Nature 317 (1985) 505 [INSPIRE].

[95] H. Murayama and J. Shu, Topological dark matter, Phys. Lett. B 686 (2010) 162 [arXiv: 0905.1720] [INSPIRE].

[96] WMAP collaboration, E. Komatsu et al., Seven-year Wilkinson Microwave Anisotropy Probe (WMAP) observations: cosmological interpretation, Astrophys. J. Suppl. 192 (2011) 18 [arXiv:1001.4538] [INSPIRE] and references therein.

[97] M. Gillioz, Dangerous skyrmions in little Higgs models, JHEP 02 (2012) 121 [arXiv:1111.2047] [INSPIRE].

[98] J.R. Ellis, V. Mayes and D.V. Nanopoulos, Flipped cryptons and the UHECRs, Phys. Rev. D 70 (2004) 075015 [hep-ph/0403144] [INSPIRE].

[99] M. Pospelov, Particle physics catalysis of thermal big bang nucleosynthesis, Phys. Rev. Lett. 98 (2007) 231301 [hep-ph/0605215] [INSPIRE].

[100] R. Foadi, M.T. Frandsen and F. Sannino, Technicolor dark matter, Phys. Rev. D 80 (2009) 037702 [arXiv:0812.3406] [INSPIRE].

[101] XENON100 collaboration, E. Aprile et al., Dark matter results from 100 live days of XENON100 data, Phys. Rev. Lett. 107 (2011) 131302 [arXiv:1104.2549] [INSPIRE] and references therein.

[102] R.J. Hill and M.P. Solon, Universal behavior in the scattering of heavy, weakly interacting dark matter on nuclear targets, Phys. Lett. B 707 (2012) 539 [arXiv:1111.0016] [INSPIRE].

[103] M.A. Shifman, A. Vainshtein and V.I. Zakharov, Remarks on Higgs boson interactions with nucleons, Phys. Lett. B 78 (1978) 443 [inSPIRE].

[104] J.R. Ellis, K.A. Olive and C. Savage, Hadronic uncertainties in the elastic scattering of supersymmetric dark matter, Phys. Rev. D 77 (2008) 065026 [arXiv:0801.3656] [INSPIRE].

[105] H. de Sandes and R. Rosenfeld, Radion-Higgs mixing effects on bounds from LHC Higgs searches, arXiv:1111.2006 [INSPIRE].

[106] V. Barger, M. Ishida and W.-Y. Keung, Dilaton at the LHC, Phys. Rev. D 85 (2012) 015024 [arXiv:1111.2580] [INSPIRE].

[107] B. Coleppa, T. Gregoire and H.E. Logan, Dilaton constraints and LHC prospects, arXiv:1111.3276 [INSPIRE]. 